\title{
Spectral analysis of the continuous and discretized heat and advection equation on single and multiple domains
}

\author{
Jens Lindström ${ }^{\star}$ and Jan Nordström ${ }^{\star \star}$ \\ *Uppsala University, Department of Information Technology, SE-751 05, Uppsala, \\ Sweden, Jens.Lindstrom@it.uu.se \\ ${ }^{\star}$ Linköping University, Department of Mathematics, SE-581 83, Linköping, \\ Sweden, Jan.Nordstrom@liu.se
}

\begin{abstract}
In this paper we study the heat and advection equation in single and multiple domains. We discretize using a second order accurate finite difference method on Summation-By-Parts form with weak boundary and interface conditions. We derive analytic expressions for the spectrum of the continuous problem and for their corresponding discretization matrices. We show how the spectrum of the single domain operator is contained in the multi domain operator spectrum when artificial interfaces are introduced. We study the impact on the spectrum and discretization errors depending on the interface treatment and verify that the results are carried over to higher order accurate schemes.
\end{abstract}

\section{Introduction}

When solving partial differential equations (PDEs) as for example the Navier-Stokes equations, there is often a need to divide the computational domain into several subdomains to allow for flexible geometry handling and sufficient resolution. The subdomains need to be coupled in a way that preserves both the stability and accuracy of the computational scheme. A proof of stability and conservation for a multiblock method for the compressible Navier-Stokes equations was shown recently in [1]. They used a finite difference method on Summation-By-Parts (SBP) form with the Simultaneous Approximation Term (SAT) technique to impose the boundary and interface conditions weakly. The SBP and SAT method has been used for many applications in fluid dynamics since it has the benefit of being provable energy stable when the correct boundary and interface conditions are imposed for the PDE $[1,2,3,4,5,6]$.

In this paper we investigate the details of the diffusion and advection operators by considering the one-dimensional heat and advection equation on single and multiple domains. The boundary and interface conditions are imposed weakly using the 
SAT technique and the equations are discretized using a second order accurate SBP operator. There are SBP operators of order 2, 3, 4 and 5 derived in for example $[7,8]$ and we stress that the stability analysis given in this paper holds for any order of accuracy. The second order operators was chosen since it allows us to derive analytical results regarding certain spectral properties of the operators.

The reason for doing such an investigation is because the SBP operators are by construction one-sided at boundaries and interfaces. The weak implementation of the boundary and interface conditions significantly modifies the scheme close to these points and we want to see exactly what influence these modifications have.

\section{Single domain spectral analysis of the heat equa- tion}

In order to compare the effects on the spectrum when introducing an artificial interface we shall begin by decomposing the heat equation on a single domain both continuously and discretized. This allows us to isolate expressions stemming from the boundaries only and separate them from the interface part.

\subsection{Continuous case}

Consider the heat equation on $-1 \leq x \leq 1$,

$$
\begin{aligned}
u_{t} & =u_{x x} \\
u(x, 0) & =f(x) \\
u(-1, t) & =g_{1}(t) \\
u(1, t) & =g_{2}(t) .
\end{aligned}
$$

To analyze (1) we introduce the Laplace transform

$$
\hat{u}=\mathcal{L} u=\int_{0}^{\infty} e^{-s t} u d t
$$

which is defined for locally integrable functions on $[0, \infty)$ where the real part of $s$ has to be sufficiently large. The basic property that we are going to use is that it transform differentiation with respect to the time variable to multiplication with the complex number $s$. Hence a time-dependent PDE in Laplace transformed space is an ordinary differential equation (ODE) which we can solve. Finding analytically the inverse transformation is in general a very difficult problem but that is not our interest here.

We shall use the Laplace transform to determine the spectrum of (1). Assume that $g_{1}=g_{2}=0$ and take the Laplace transform of (1). The initial condition is omitted since it does not enter in the spectral analysis. We get an ODE in transformed space,

$$
\begin{aligned}
s \hat{u} & =\hat{u}_{x x} \\
\hat{u}(-1, s) & =0 \\
\hat{u}(1, s) & =0,
\end{aligned}
$$


which is an eigenvalue problem for $s$. By the ansatz $\hat{u}=e^{k x}$ we can determine that the general solution to $(3)$ is

$$
\hat{u}=c_{1} e^{\sqrt{s} x}+c_{2} e^{-\sqrt{s} x} .
$$

By applying the boundary conditions we obtain

$$
\begin{aligned}
& c_{1} e^{-\sqrt{s}}+c_{2} e^{\sqrt{s}}=0 \\
& c_{1} e^{\sqrt{s}}+c_{2} e^{-\sqrt{s}}=0
\end{aligned}
$$

which we write in matrix form as

$$
\underbrace{\left[\begin{array}{cc}
e^{-\sqrt{s}} & e^{\sqrt{s}} \\
e^{\sqrt{s}} & e^{-\sqrt{s}}
\end{array}\right]}_{E(s)}\left[\begin{array}{l}
c_{1} \\
c_{2}
\end{array}\right]=\left[\begin{array}{l}
0 \\
0
\end{array}\right] .
$$

Equation (7) will have a non-trivial solution when the coefficient matrix $E(s)$ is singular. We hence seek the values of $s$ such that the determinant is zero. We have

$$
\operatorname{det}(E(s))=-2 \sinh (2 \sqrt{s})
$$

which is zero for

$$
s=-\frac{\pi^{2} n^{2}}{4}, \quad n \in \mathbb{N}
$$

This infinite sequence of values is thus the spectrum of (1). Note that $s=0$ is not considered a solution since then we have a double root and $\hat{u}=c_{1}+c_{2} x$. From the boundary conditions we get that $\hat{u} \equiv 0$ and hence $u \equiv 0$, which is trivial.

Remark 2.1. Note that the spectrum is identical to that obtained by standard SturmLiouville theory but with the sign reversed. From the Gustafsson-Kreiss-Sundström theory point of view, the negative sign implies that (1) is well-posed [9].

\subsection{Discrete case}

To discretize (1) we use a second order accurate finite difference operator on SBP form,

$$
u_{x x} \approx D_{2} v
$$

where $v=\left[v_{0}, v_{1}, \ldots, v_{N}\right]^{T}$ is the discrete grid function and the mesh is uniform with $N+1$ grid points. The exact form of the operator $D_{2}$ is, see $[7,10]$,

$$
D_{2}=P^{-1}(-A+B D)=\frac{1}{\Delta x^{2}}\left[\begin{array}{cccccc}
0 & 0 & 0 & 0 & \cdots & 0 \\
1 & -2 & 1 & 0 & \cdots & 0 \\
\vdots & \ddots & \ddots & \ddots & \ddots & \vdots \\
0 & \cdots & 0 & 1 & -2 & 1 \\
0 & 0 & 0 & 0 & \cdots & 0
\end{array}\right]
$$


where

$$
\begin{gathered}
P=\Delta x\left[\begin{array}{ccccc}
\frac{1}{2} & 0 & 0 & \cdots & 0 \\
0 & 1 & 0 & \cdots & 0 \\
\vdots & \ddots & \ddots & \ddots & \vdots \\
0 & \cdots & 0 & 1 & 0 \\
0 & \cdots & 0 & 0 & \frac{1}{2}
\end{array}\right], A=\frac{1}{\Delta x}\left[\begin{array}{ccccc}
1 & -1 & 0 & \cdots & 0 \\
-1 & 2 & -1 & \cdots & 0 \\
\vdots & \ddots & \ddots & \ddots & \vdots \\
0 & \cdots & -1 & 2 & -1 \\
0 & \cdots & 0 & -1 & 1
\end{array}\right] \\
B=\left[\begin{array}{cccccc}
-1 & 0 & 0 & \cdots & 0 \\
0 & 0 & 0 & \cdots & 0 \\
\vdots & \ddots & \ddots & \ddots & \vdots \\
0 & \cdots & 0 & 0 & 0 \\
0 & \cdots & 0 & 0 & 1
\end{array}\right], D=\frac{1}{\Delta x}\left[\begin{array}{ccccc}
-1 & 1 & \cdots & 0 & 0 \\
-\frac{1}{2} & 0 & \frac{1}{2} & \cdots & 0 \\
\vdots & \ddots & \ddots & \ddots & \vdots \\
0 & 0 & -\frac{1}{2} & 0 & \frac{1}{2} \\
0 & 0 & \cdots & -1 & 1
\end{array}\right]
\end{gathered}
$$

Note that (11) has zeros on the top and bottom row and is hence inconsistent at the boundaries. This does however not affect the global accuracy because of the SAT implementation of the weak boundary conditions $[7,11,12]$.

The entire scheme for (1) can be written as

$$
v_{t}=D_{2} v+\sigma_{1} P^{-1} D^{T} e_{0}\left(v_{0}-g_{1}\right)+\sigma_{2} P^{-1} D^{T} e_{N}\left(v_{N}-g_{2}\right)
$$

where $P$ is the positive symmetric matrix in (12) which defines a discrete norm by $\|w\|^{2}=w^{T} P w$. The vectors $e_{0, N}$ are zero vectors except for the first and last position respectively, which is one. The two parameters $\sigma_{1,2}$ will be determined such that the scheme is stable in the $P$-norm $[8,13]$.

\subsubsection{Stability}

We use the energy method to determine the coefficients $\sigma_{1,2}$ such that the scheme is stable. The stability of the scheme ensures that all eigenvalues of the complete difference operator, including the boundary conditions, have non-positive real parts.

By multiplying (13) by $v^{T} P$ and adding the transpose to itself we obtain

$$
\|v\|_{t}^{2}=2\left(\sigma_{1}-1\right) v_{0}(D v)_{0}+2\left(\sigma_{2}+1\right) v_{N}(D v)_{N}-v^{T}\left(A+A^{T}\right) v
$$

It is clear that the scheme is stable if we choose

$$
\sigma_{1}=1, \quad \sigma_{2}=-1
$$

since the last term in (14) is dissipative.

\subsubsection{Complete eigenspectrum}

Consider (13) again with homogeneous boundary conditions. Since we have kept time continuous we can take the Laplace transform of the entire scheme and after rearranging we get

$$
\underbrace{\left(s I-D_{2}-\sigma_{1} P^{-1} D^{T} E_{0}-\sigma_{2} P^{-1} D^{T} E_{N}\right)}_{M} \hat{v}=0
$$


where $I$ is the $N+1$ dimensional identity operator and $E_{0, N}$ are zero matrices except for the $(0,0)$ and $(N, N)$ positions respectively which is one. To determine the complete eigenspectrum of the discrete operator $M$ we start by considering the difference scheme for an internal point. The internal scheme is the standard central finite difference scheme and hence

$$
\frac{\partial}{\partial t} v_{i}=\frac{v_{i-1}-2 v_{i}+v_{i+1}}{\Delta x^{2}}
$$

By taking the Laplace transform of (17) we obtain a recurrence relation

$$
s \hat{v}_{i}=\frac{\hat{v}_{i-1}-2 \hat{v}_{i}+\hat{v}_{i+1}}{\Delta x^{2}}
$$

for which we can obtain the general solution by the ansatz $\hat{v}_{i}=\sigma \kappa^{i}$. The ansatz yields the second order equation

$$
\kappa^{2}-(\tilde{s}+2) \kappa+1=0
$$

with the two solutions

$$
\kappa_{+,-}=\frac{\tilde{s}+2}{2} \pm \sqrt{\left(\frac{\tilde{s}+2}{2}\right)^{2}-1}
$$

where $\tilde{s}=s \Delta x^{2}$. Hence the general solution to (18) is

$$
\hat{v}_{i}=c_{1} \kappa_{+}^{i}+c_{2} \kappa_{-}^{i} \text {. }
$$

To obtain the eigenspectrum of $M$ we consider the boundary points. The scheme is modified at grid points $x_{0}, x_{1}, x_{N-1}$ and $x_{N}$ and the corresponding equations are after substituting (15)

$$
\begin{aligned}
(\tilde{s}+2) \hat{v}_{0} & =0 \\
-2 \hat{v}_{0}+(\tilde{s}+2) \hat{v}_{1}-\hat{v}_{2} & =0 \\
-\hat{v}_{N-2}+(\tilde{s}+2) \hat{v}_{N-1}-2 \hat{v}_{N} & =0 \\
(\tilde{s}+2) \hat{v}_{N} & =0 .
\end{aligned}
$$

If we assume that the ansatz (21) is valid at gridpoints $x_{i}, i=1, \ldots, N-1$ we get by substituting (21) into (22) the square matrix equation

$$
\underbrace{\left[\begin{array}{cccc}
\tilde{s}+2 & 0 & 0 & 0 \\
-2 & \left((\tilde{s}+2)-\kappa_{+}\right) \kappa_{+} & \left((\tilde{s}+2)-\kappa_{-}\right) \kappa_{-} & 0 \\
0 & \left((\tilde{s}+2) \kappa_{+}-1\right) \kappa_{+}^{N-2} & \left((\tilde{s}+2) \kappa_{-}-1\right) \kappa_{-}^{N-2} & -2 \\
0 & 0 & 0 & \tilde{s}+2
\end{array}\right]}_{E(s, \kappa)}\left[\begin{array}{c}
\hat{v}_{0} \\
c_{1} \\
c_{2} \\
\hat{v}_{N}
\end{array}\right]=\left[\begin{array}{l}
0 \\
0 \\
0 \\
0
\end{array}\right]
$$

Equation (23) will have a non-trivial solution for the values of $\tilde{s}$ which makes $E(s, \kappa)$ singular. Thus we seek the values of $\tilde{s}$ for which $\operatorname{det}(E(s, \kappa))=0$. These values of $\tilde{s}$ constitute the spectrum of $M[9]$. The determinant of $E(s, \kappa)$ is

$$
\operatorname{det}(E(s, \kappa))=(\tilde{s}+2)^{2}\left(\kappa_{-}^{N}-\kappa_{+}^{N}\right)
$$


and we can see that the spectrum containts the points for which

$$
\tilde{s}=-2, \quad \kappa_{+}^{N}=\kappa_{-}^{N} .
$$

In the second case we let $\kappa_{+}=a e^{i \theta}, \kappa_{-}=b e^{i \phi}$ and we can by identifying the radius and argument determine that

$$
\tilde{s}=2\left((-1)^{k} \cos \left(\frac{\pi k}{N}\right)-1\right), \quad k=1, \ldots, N-1 .
$$

Thus we have found $N+1$ values of $\tilde{s}$ which gives non-trivial solutions to (23) and hence they constitute the entire spectrum of $M$. To verify we compare by numerically computing the eigenvalues of $\Delta x^{2} M$ with $N=16$ which is seen in Figure 1.

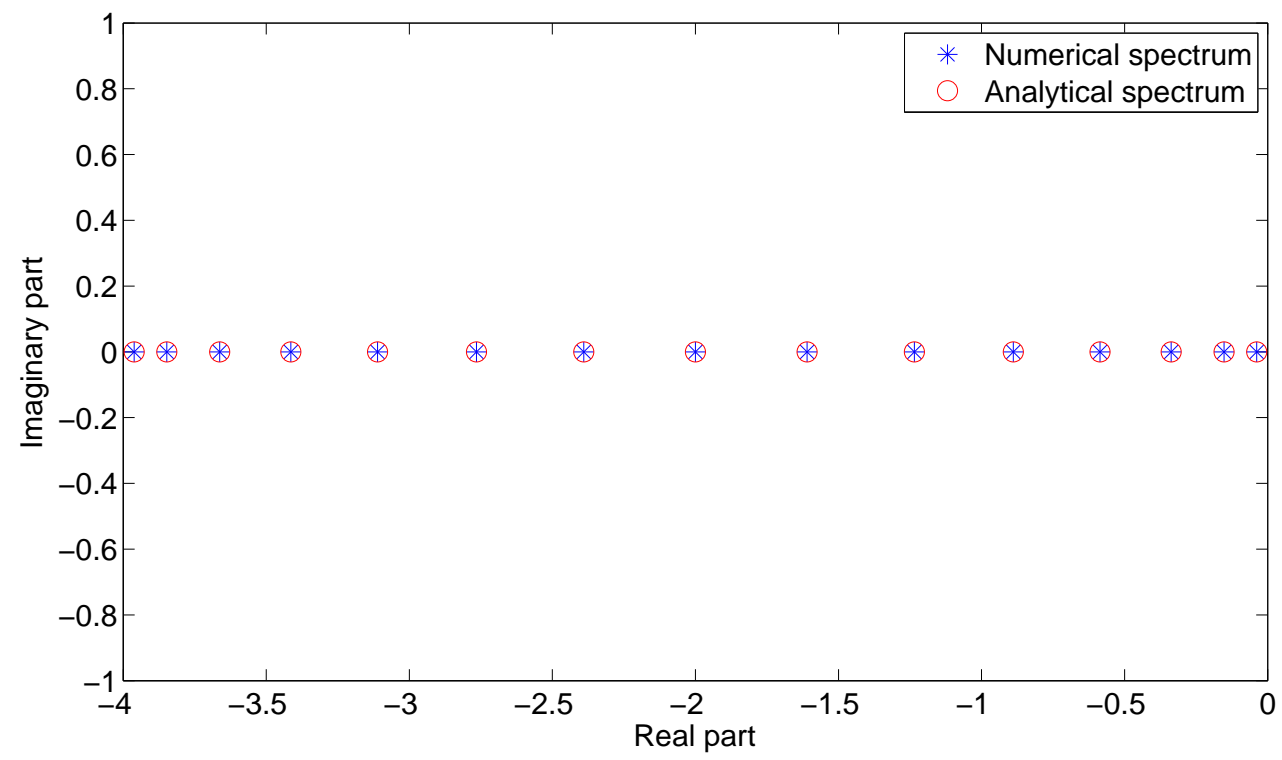

Figure 1: Analytical and computed numerical spectrum. There is a tripple root at $\tilde{s}=-2$.

Remark 2.2. One has to be careful with double roots. From (20) and (24) a possible solution would be when

$$
\left(\frac{\tilde{s}+2}{2}\right)^{2}-1=0
$$

or equivalently $\tilde{s}=-4$ or $\tilde{s}=0$. These are however false roots. A proof is given in Appendix A. Interesting is though that all eigenvalues of $E(s, \kappa)$ are contained between $\tilde{s}=-4$ and $\tilde{s}=0$.

\subsubsection{Convergence of eigenvalues}

To see how the eigenvalues of the discretization matrix converge to the eigenvalues of the continuous PDE, we let (9) be denoted by $\mu_{n}$. We rescale (26) with $\Delta x^{2}$ and denote it by $\tilde{\lambda}_{k}$. Since $\Delta x=\frac{2}{N}$ we can rewrite $\tilde{\lambda}_{k}$ as

$$
\lambda_{n}=\frac{N^{2}}{2}\left(\cos \left(\frac{\pi n}{N}\right)-1\right), \quad n=1, \ldots, N-1
$$


which generates the same sequence as (26), but it is monotonically decreasing. This allows us to compare $\mu_{n}$ and $\lambda_{n}$ elementwise.

By assuming that $n<N$ we can Taylor expand (28) around zero and simplify to get

$$
\lambda_{n}=\mu_{n}+O\left(\frac{n^{4}}{N^{2}}\right) .
$$

We can see that for large $N$ we can expect the first $\sqrt{N}$ eigenvalues to be rather well approximated while for larger $n$ than that, they will start to diverge. This is the typical situation. When the resolution is increased, more eigenvalues will be converged but eigenvalues that are not converging will be created.

\section{Multi domain spectral analysis of the heat equa- tion}

In this section we shall use the knowledge obtained in the previous section to determine spectral properties when an artificial interface has been introduced in the domain. Our goal is to determine how the introduction of an interface influences the spectrum of both the continuous and discrete equations. Moreover we want to design the interface treatment in such a way that it is similar to, or maybe even better than, the single domain spectrum if possible.

\subsection{Continuous case}

Consider now two heat equations coupled over an interface at $x=0$ with homogeneous boundary conditions

$$
\begin{aligned}
u_{t} & =u_{x x}, \quad-1 \leq x \leq 0, \\
v_{t} & =v_{x x}, \quad 0 \leq x \leq 1, \\
u(-1, t) & =0 \\
v(1, t) & =0, \\
u(0, t)-v(0, t) & =0, \\
u_{x}(0, t)-v_{x}(0, t) & =0 .
\end{aligned}
$$

We take the Laplace transform again as before and obtain the general solutions for $\hat{u}$ and $\hat{v}$ as

$$
\begin{gathered}
\hat{u}=c_{1} e^{\sqrt{s} x}+c_{2} e^{-\sqrt{s} x} \\
\hat{v}=c_{3} e^{\sqrt{s} x}+c_{4} e^{-\sqrt{s} x}
\end{gathered}
$$

By applying the boundary and interface conditions we get the matrix equation

$$
\underbrace{\left[\begin{array}{cccc}
e^{-\sqrt{s}} & e^{\sqrt{s}} & 0 & 0 \\
1 & 1 & -1 & -1 \\
1 & -1 & -1 & 1 \\
0 & 0 & e^{\sqrt{s}} & e^{-\sqrt{s}}
\end{array}\right]}_{E(s)}\left[\begin{array}{l}
c_{1} \\
c_{2} \\
c_{3} \\
c_{4}
\end{array}\right]=\left[\begin{array}{l}
0 \\
0 \\
0 \\
0
\end{array}\right]
$$


with a non-trivial solution when $\operatorname{det}(E(s))=0$. A direct computation of the determinant shows that $\operatorname{det}(E(s))=4 \sinh (2 \sqrt{s})$ and hence the spectrum remains unchanged by the introduction of an interface. This is of course all in order since the interface is purely artificial. However when we discretize (30) we modify the scheme at the interface and we can expect that this modification will influence the eigenvalues of the complete difference operator.

\section{$3.2 \quad$ Discrete case}

In order to proceed we assume that there are equally many grid points in each subdomain and that the grid spacing is the same. This means that we can apply the same operators in both domains which will simplify the notation and algebra.

With a slight abuse of notation we now let $u$ and $v$ denote the discrete grid functions with both having $N+1$ components. Thus there are in total $2 N+2$ grid points in the domain since the interface point occurs twice, and the resolution is twice as high as in the single domain case.

By using the SBP and SAT technique we can discretize (30) as

$$
\begin{aligned}
u_{t} & =D_{2} u+\sigma_{1} P^{-1} D^{T} E_{0} u \\
& +\sigma_{2} P^{-1} D^{T} e_{N}\left(u_{N}-v_{0}\right)+\sigma_{3} P^{-1} e_{N}\left((D u)_{N}-(D v)_{0}\right) \\
v_{t} & =D_{2} v+\tau_{1} P^{-1} D^{T} E_{N} v \\
& +\tau_{2} P^{-1} D^{T} e_{0}\left(v_{0}-u_{N}\right)+\tau_{3} P^{-1} e_{0}\left((D v)_{0}-(D u)_{N}\right)
\end{aligned}
$$

The unknown penalty parameters $\sigma_{1,2,3}$ and $\tau_{1,2,3}$ has again to be determined for stability.

\subsubsection{Stability}

To determine the unknown parameters $\sigma_{1,2,3}$ and $\tau_{1,2,3}$ we multiply the first equation in (33) with $u^{T} P$ and the second with $v^{T} P$. We add the transposes of the resulting expressions to themselves to get

$$
\begin{aligned}
\|u\|_{t}^{2} & =-2 u_{0}(D u)_{0}+2 u_{N}(D u)_{N}-u^{T}\left(A+A^{T}\right) u \\
& +2 \sigma_{1}(D u)_{0} u_{0}+2 \sigma_{2}(D u)_{N}\left(u_{N}-v_{0}\right)+2 \sigma_{3} u_{M}\left((D u)_{N}-(D v)_{0}\right) \\
\|v\|_{t}^{2} & =-2 v_{0}(D v)_{0}+2 v_{N}(D v)_{N}-v^{T}\left(A+A^{T}\right) v \\
& +2 \tau_{1}(D v)_{N} v_{N}+2 \tau_{2}(D v)_{0}\left(v_{0}-u_{N}\right)+2 \tau_{3} v_{0}\left((D v)_{0}-(D u)_{N}\right) .
\end{aligned}
$$

By adding both expressions in (34) we can write the result as

$$
\begin{aligned}
\|u\|_{t}^{2}+\|v\|_{t}^{2} & =2\left(\sigma_{1}-1\right) u_{0}(D u)_{0}+2\left(\tau_{1}+1\right) v_{N}(D v)_{N} \\
& +q^{T} H q-u^{T}\left(A+A^{T}\right) u-v^{T}\left(A+A^{T}\right) v
\end{aligned}
$$

where $q=\left[u_{N},(D u)_{N}, v_{0},(D v)_{0}\right]^{T}$ and

$$
H=\left[\begin{array}{cccc}
0 & 1+\sigma_{2}+\sigma_{3} & 0 & -\left(\tau_{2}+\tau_{3}\right) \\
1+\sigma_{2}+\sigma_{3} & 0 & -\left(\tau_{2}+\tau_{3}\right) & 0 \\
0 & -\left(\sigma_{2}+\tau_{3}\right) & 0 & -1+\tau_{2}+\tau_{3} \\
-\left(\sigma_{3}+\tau_{2}\right) & 0 & -1+\tau_{2}+\tau_{3} & 0
\end{array}\right]
$$


In order to bound (35) we have to choose

$$
\sigma_{1}=1, \quad \tau_{1}=-1
$$

as in the single domain case, and we have to choose the rest of the penalty parameters such that $H \leq 0$. This is easily accomplished by noting that the diagonal of $H$ consists of zeros only, and hence by the Gershgorin theorem we need to put all remaining entires to zero to ensure the semidefiniteness of $H$. This gives us a oneparameter family of solutions

$$
r \in \mathbb{R}, \quad \sigma_{2}=-(1+r), \quad \sigma_{3}=r, \quad \tau_{2}=-r, \quad \tau_{3}=1+r .
$$

Thus all penalty parameters have been determined and the scheme is stable.

Worth noting is that the parameter $r$ determines how the equations are coupled. For $r=0$ two of the penalty parameters in (38) disappear and renders the scheme one-sided coupled in the sense that the left domain receives a solution value from the right domain and gives the value of its gradient to the right domain. For $r=-1$ the situation is reversed and for other values of $r$, the scheme is fully coupled. Note that the scheme is stable for all choices of $r$. We shall investigate the influence of the interface paramater in later sections. More details can also be found in [6].

\subsubsection{Eigenspectrum}

The scheme (33) with a second order accurate difference operator makes eight grid points (two at each boundary and four at the interface) stray from a standard central finite difference scheme. This is a significant modification and we can expect that there will be a global impact depending on these modifications. A direct way of investigating this is by considering the change on the spectrum due to the modifications.

We take the Laplace transform of (33) and consider the difference equations at the modified boundary and interface points. We get after substituting (37) and (38) into (33) that

$$
\begin{aligned}
(\tilde{s}+2) \hat{u}_{0} & =0 \\
-2 \hat{u}_{0}+(\tilde{s}+2) \hat{u}_{1}-\hat{u}_{2} & =0 \\
-\hat{u}_{N-2}+(\tilde{s}+2) \hat{u}_{N-1}-(2+r) \hat{u}_{N}+(1+r) \hat{v}_{0} & =0 \\
2 r \hat{u}_{N-1}+(\tilde{s}+2) \hat{u}_{N}-2(1+2 r) \hat{v}_{0}+2 r \hat{v}_{1} & =0 \\
-2(1+r) \hat{u}_{N-1}+2(1+2 r) \hat{u}_{N}+(\tilde{s}+2) \hat{v}_{0}-2(1+r) \hat{v}_{1} & =0 \\
-r \hat{u}_{N}-(1-r) \hat{v}_{0}+(\tilde{s}+2) \hat{v}_{1}-\hat{v}_{2} & =0 \\
-\hat{v}_{N-2}+(\tilde{s}+2) \hat{v}_{N-1}-2 \hat{v}_{N} & =0 \\
(\tilde{s}+2) \hat{v}_{N} & =0 .
\end{aligned}
$$

From the internal schemes we have similarly as before that

$$
\begin{aligned}
& \hat{u}_{i}=c_{1} \kappa_{+}^{i}+c_{2} \kappa_{-}^{i} \\
& \hat{v}_{j}=c_{3} \kappa_{+}^{j}+c_{4} \kappa_{-}^{j}
\end{aligned}
$$


where $\kappa_{+,-}$are the same as in $(20)$ and $i, j=1, \ldots, N-1$. By substituting (40) into (39) we get the matrix equation $E(r, s, \kappa) w=0$ for the unknowns

$$
w=\left[\hat{u}_{0}, c_{1}, c_{2}, \hat{u}_{N}, \hat{v}_{0}, c_{3}, c_{4}, \hat{v}_{N}\right]^{T}
$$

where

$$
E(r, s, \kappa)=\left[\begin{array}{cccccccc}
\tilde{s}+2 & 0 & 0 & 0 & 0 & 0 & 0 & 0 \\
-2 & 1 & 1 & 0 & 0 & 0 & 0 & 0 \\
0 & e_{3,2} & e_{3,3} & e_{3,4} & e_{3,5} & e_{3,6} & e_{3,7} & 0 \\
0 & e_{4,2} & e_{4,3} & e_{4,4} & e_{4,5} & e_{4,6} & e_{4,7} & 0 \\
0 & e_{5,2} & e_{5,3} & e_{5,4} & e_{5,5} & e_{5,6} & e_{5,7} & 0 \\
0 & e_{6,2} & e_{6,3} & e_{6,4} & e_{6,5} & e_{6,6} & e_{6,7} & 0 \\
0 & 0 & 0 & 0 & 0 & \kappa_{+}^{N} & \kappa_{-}^{N} & -2 \\
0 & 0 & 0 & 0 & 0 & 0 & 0 & \tilde{s}+2
\end{array}\right]
$$

with coefficients $e_{i, j}$ given by

$$
\begin{array}{lll}
e_{3,2}=\kappa_{+}^{N} & e_{3,3}=\kappa_{-}^{N} & e_{3,4}=-(2+r) \\
e_{3,5}=1+r & e_{3,6}=0 & e_{3,7}=0 \\
e_{4,2}=2 r \kappa_{+}^{N-1} & e_{4,3}=2 r \kappa_{-}^{N-1} & e_{4,4}=\tilde{s}+2 \\
e_{4,5}=-2(1+2 r) & e_{4,6}=2 r \kappa_{+} & e_{4,7}=2 r \kappa_{-} \\
e_{5,2}=-2(1+r) \kappa_{+}^{N-1} & e_{5,3}=-2(1+r) \kappa_{-}^{N-1} & e_{5,4}=2(1+2 r) \\
e_{5,5}=\tilde{s}+2 & e_{5,6}=-2(1+r) \kappa_{+} & e_{5,7}=-2(1+r) \kappa_{-} \\
e_{6,2}=0 & e_{6,3}=0 & e_{6,4}=-r \\
e_{6,5}=-(1-r) & e_{6,6}=1 & e_{6,7}=1 .
\end{array}
$$

As before we obtain the spectrum by computing all values of $\tilde{s}$ such that $\operatorname{det}(E(r, s, \kappa))=$ 0 . It is easy to see by expanding the determinant by the first and last row that

$$
\operatorname{det}\left(E(r, s, \kappa)=-(\tilde{s}+2)^{2} \operatorname{det}(\tilde{E}(r, s, \kappa))\right.
$$

where $\tilde{E}(r, s, \kappa)$ is the inner $6 \times 6$ matrix. The determinant of $\tilde{E}(r, s, \kappa)$ is somewhat more complicated but by expanding it further and factorizing we get

$$
\operatorname{det}\left(E(r, s, \kappa)=(\tilde{s}+2)\left(\kappa_{-}^{N}-\kappa_{+}^{N}\right) f(r, s, \kappa) .\right.
$$

We can see that the two first factors in (45) are exactly (24). Thus the spectrum from the single domain operator is contained in the multi domain operator spectrum. This is visualized in Figure 2. The last factor $f(r, s, \kappa)$ is given explicitly by

$$
\begin{aligned}
f(r, s, \kappa) & =\left(16 r^{2}+16 r+\tilde{s}^{2}+4 \tilde{s}+8\right)\left(\kappa_{-}^{N}-\kappa_{+}^{N}\right) \\
& +2\left(8 r^{3}+12 r^{2}+2 r \tilde{s}+8 r+\tilde{s}+2\right)\left(\kappa_{+}^{N} \kappa_{-}-\kappa_{+} \kappa_{-}^{N}\right) \\
& +2\left(2 r^{2} \tilde{s}-4 r^{2}+4 r \tilde{s}-4 r+\tilde{s}-2\right)\left(\kappa_{-}^{N-1}-\kappa_{-}^{N-1}\right) .
\end{aligned}
$$

A closed form for the zeros of (46) have not been found. However, we can numerically compute the zeros. 


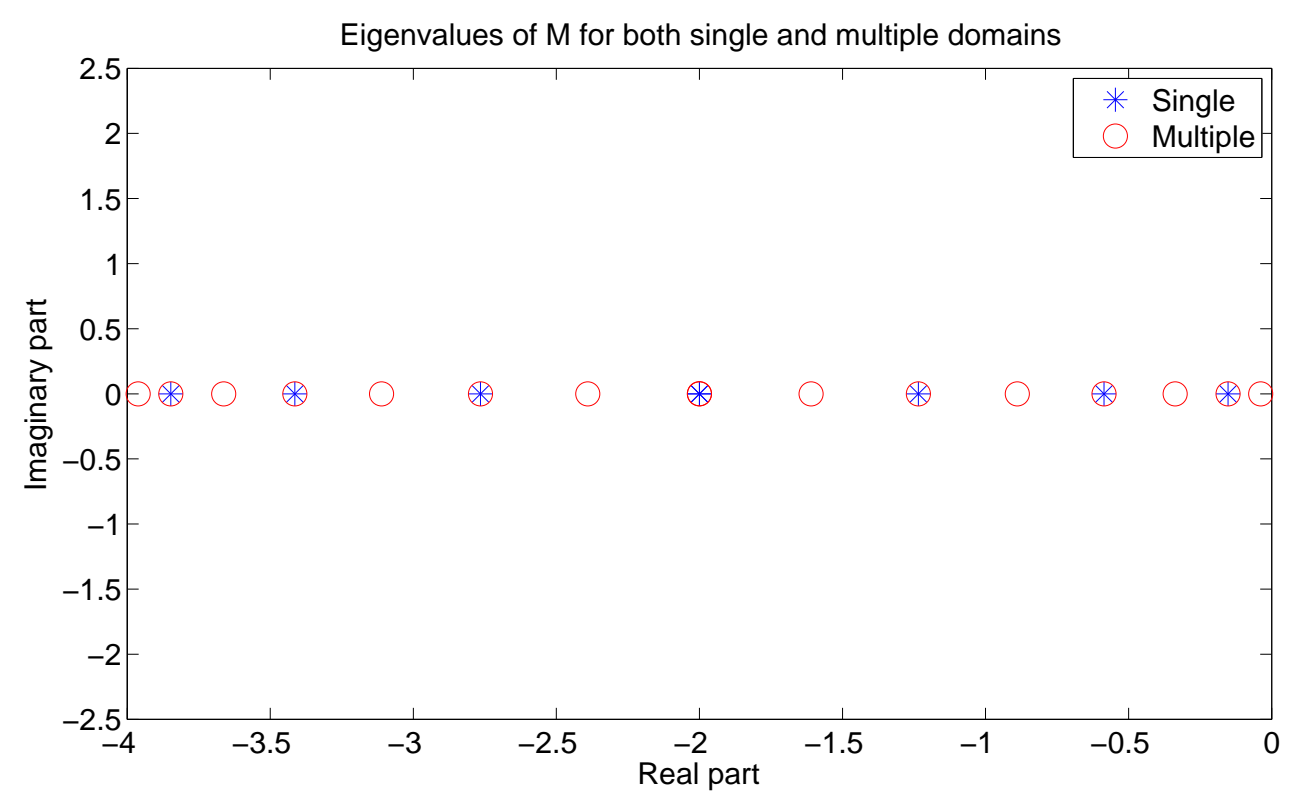

Figure 2: Eigenvalues of the single domain operator with 9 grid points and the multi domain operator with 18 grid points scaled with $\Delta x^{2}$. The single domain operator spectrum is always contained in the multi domain operator spectrum independent of the interface parameter $r$. There is a tripple root at $\tilde{s}=-2$.

\subsection{Influence of the type of coupling}

The type of coupling depends on the interface parameter $r$ in (38) and by varying it, the spectral properties are modified. The interface parameter can be considered as a weight between Dirichlet and Neumann conditions. When $r=0$ or $r=-1$, some of the terms in (38) are cancelled and renders the scheme one-sided coupled in the sense that one domain gives its value to the other domain and receives the value of the gradient. Since the extremal values are $r=-1,0$ one might expect that something interesting happens when $r=-\frac{1}{2}$, that is when the two equations are coupled symmetrically. The case $r=-\frac{1}{2}$ will be denoted by the symmetric coupling and all other cases by unsymmetric coupling.

By considering the equations that are modified at the interface,

$$
\begin{aligned}
\frac{\partial}{\partial t} u_{N-1} & =\frac{u_{N-2}-2 u_{N-1}+(2+r) u_{N}-(1+r) v_{0}}{\Delta x^{2}} \\
\frac{\partial}{\partial t} u_{N} & =\frac{-2 r u_{N-1}-2 u_{N}+2(1+2 r) v_{0}-2 r v_{1}}{\Delta x^{2}} \\
\frac{\partial}{\partial t} v_{0} & =\frac{2(1+r) u_{N-1}-2(1+2 r) u_{N}-2 v_{0}+2(1+r) v_{1}}{\Delta x^{2}} \\
\frac{\partial}{\partial t} v_{1} & =\frac{r u_{N}+(1-r) v_{0}-2 v_{1}+v_{2}}{\Delta x^{2}}
\end{aligned}
$$

we can easily see how the difference scheme is modified due to the choice of $r$.

By taking an exact solution $w(x, t)$ to (30) we can by Taylor expanding (47) determine the accuracy. To simplify the notation we drop the indicies and expand 
all equations around $x_{j}=x_{*}$. We get

$$
\begin{aligned}
& \frac{\partial}{\partial t} w\left(x_{*}, t\right)=w_{x x}\left(x_{*}, t\right)+O\left(\Delta x^{2}\right) \\
& \frac{\partial}{\partial t} w\left(x_{*}, t\right)=-2 r w_{x x}\left(x_{*}, t\right)+O\left(\Delta x^{2}\right) \\
& \frac{\partial}{\partial t} w\left(x_{*}, t\right)=2(1+r) w_{x x}\left(x_{*}, t\right)+O\left(\Delta x^{2}\right) \\
& \frac{\partial}{\partial t} w\left(x_{*}, t\right)=w_{x x}\left(x_{*}, t\right)+O\left(\Delta x^{2}\right)
\end{aligned}
$$

for the corresponding equations in (47). We can now easily see that we obtain the second order accurate second derivative only for $r=-\frac{1}{2}$. Even though some of the above equations correspond to inconsistent approximations of the second derivative, the global accuracy of the operator remain unchanged [7, 13, 12].

The similarity with the standard second order SBP scheme is immediately seen when considering the eigenvalues of the complete operator $M$. Figure 3 and Figure 4 shows the eigenvalues of $M$ scaled with $\Delta x^{2}$ of both the single and multi domain operator for different values of $r$. The subdomains are using 9 grid points each to a total number of 18 while the single domain is using 17 grid points. Note in

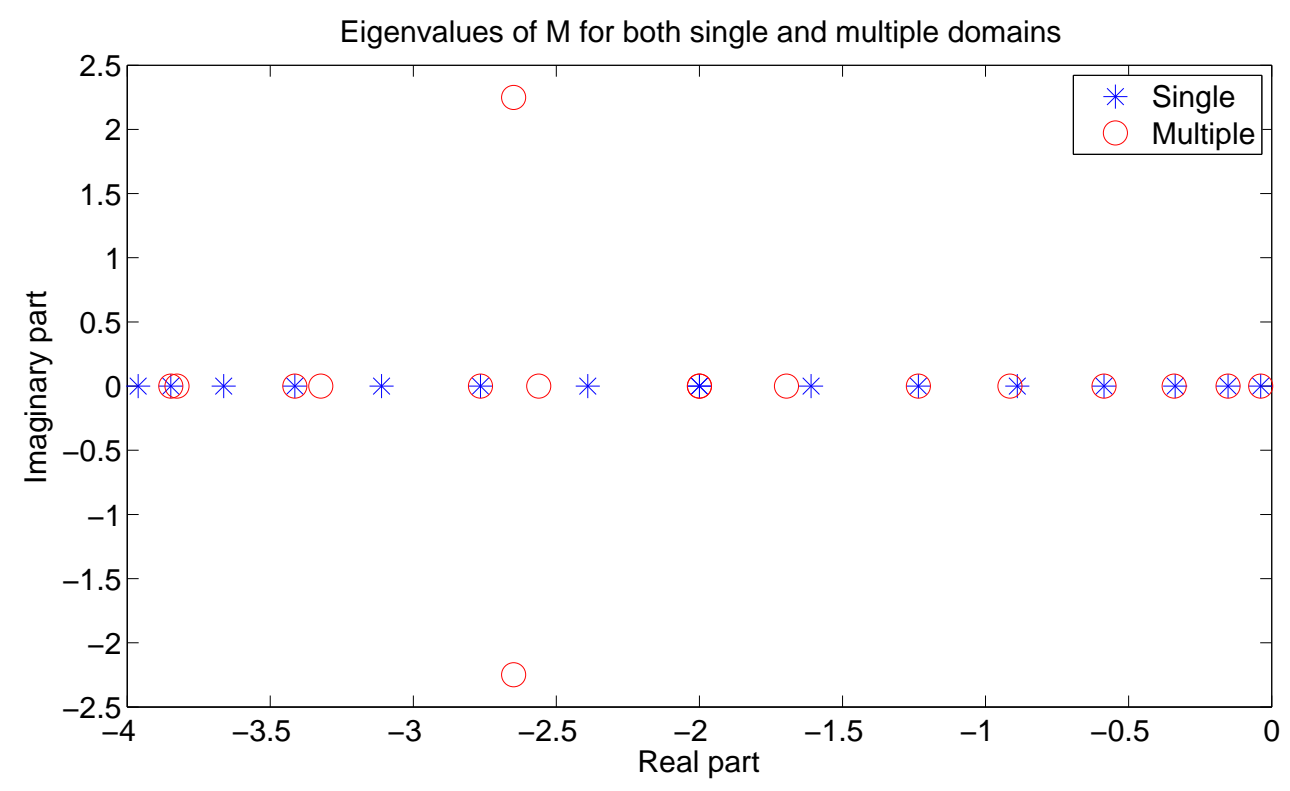

Figure 3: Eigenvalues of the single domain operator with 17 grid points and the multi domain operator with 18 grid points scaled with $\Delta x^{2} \cdot r=0$. All eigenvalues are identical if using $r=-1$.

Figure 3 that there is a complex conjugate pair of eigenvalues. The magnitude of this pair increases with increasing magnitude of $r$ and hence potentially makes the discretization more stiff.

\subsubsection{Stiffness and convergence to steady-state}

To see how the stiffness is affected by the interface treatment we plot the largest absolute value of the eigenvalues of the discretization matrix as a function of $r$ in 


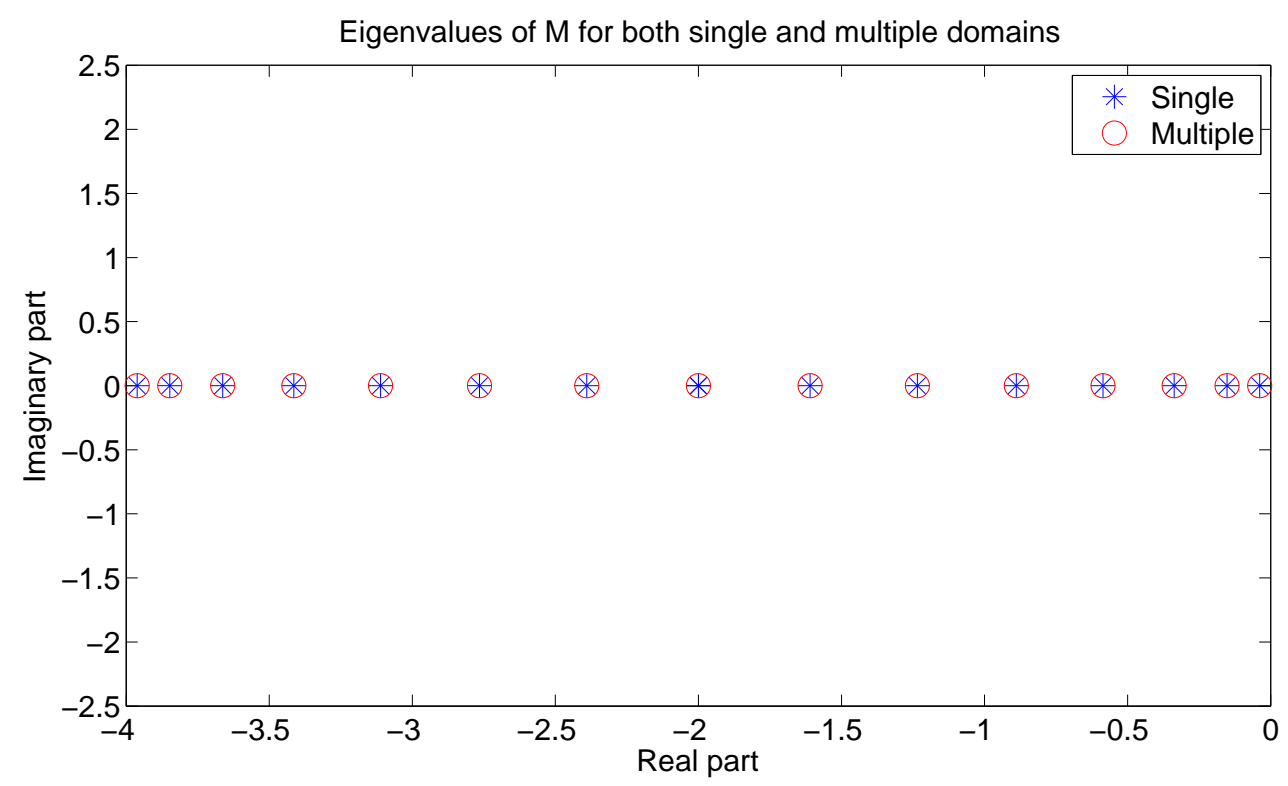

Figure 4: Eigenvalues of the single domain operator with 17 grid points and the multi domain operator with 18 grid points scaled with $\Delta x^{2} \cdot r=-\frac{1}{2}$.

Figure 5. We can see that with increasing magnitue of $r$, the discretization become more stiff as expected. More unexpected is that the stiffness is slightly reduced below that of the uncoupled equations by choosing an unsymmetric coupling. This is contrary to the result in [6]. However in that paper a wide operator was used which have a different set of eigenvalues.

It is beneficial for the rate of convergence to steady-state with a discretization which have its real parts of the spectrum bounded away from zero as far as possible $[5,14,15,16]$. In Figure 6 we show the real part of the spectrum closest to zero as a function of $r$.

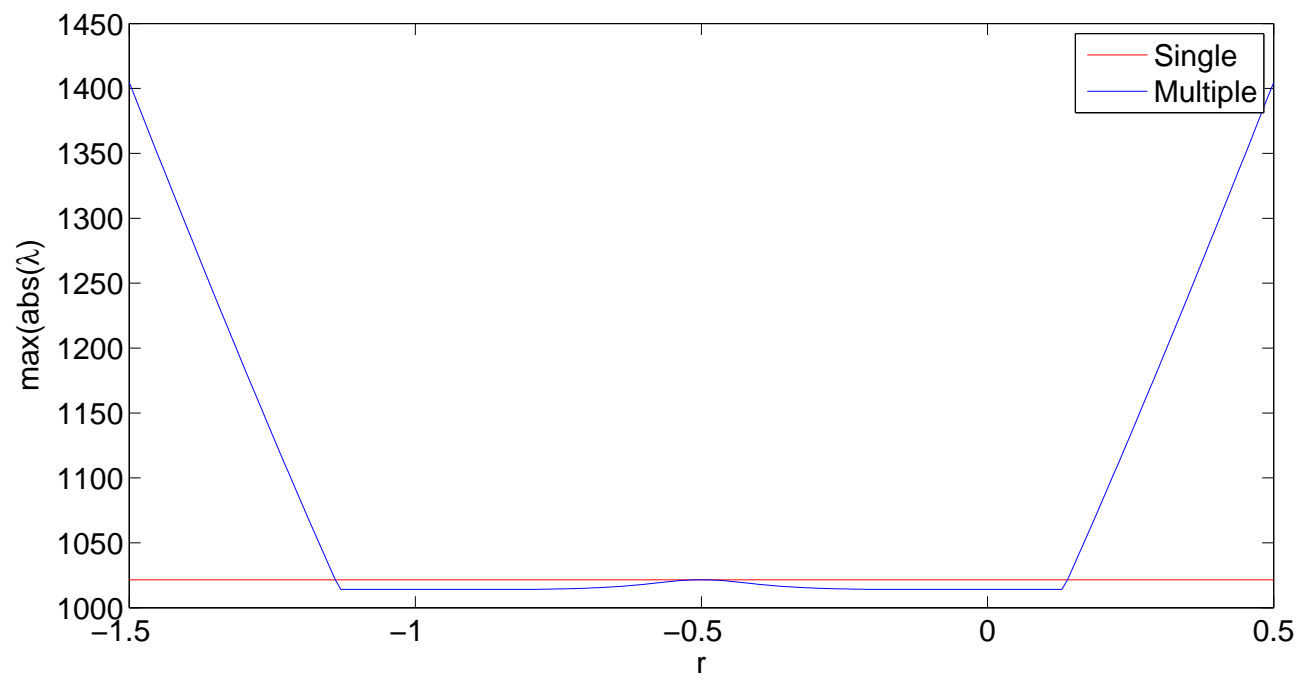

Figure 5: $\max (\operatorname{abs}(\lambda))$ as function of $r$. The single domain operator is using 33 grid points and the multi domain operator is using 34 grid points. 


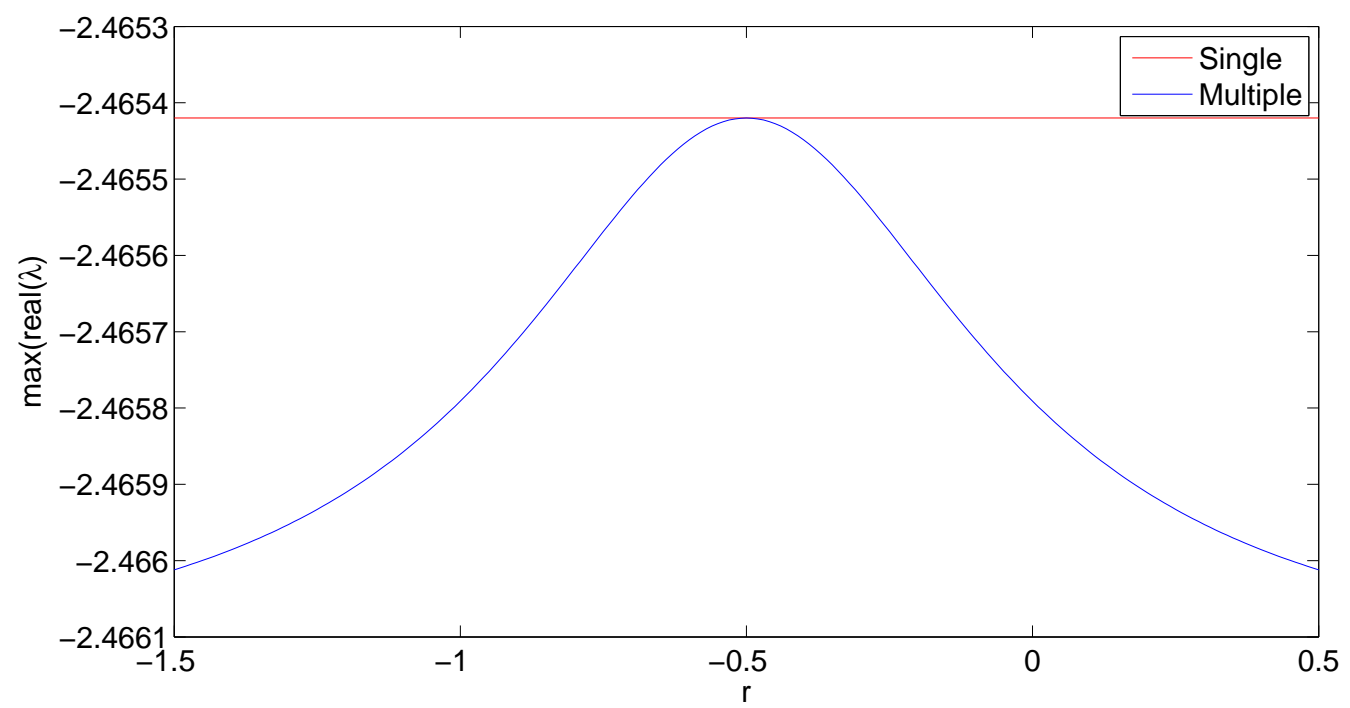

Figure 6: $\max (\mathbb{R}(\lambda))$ as a function of $r$. The single domain operator is using 33 grid points and the multi domain operator is using 34 grid points.

We have used 33 grid points for the single domain in both Figure 5 and Figure 6 and hence the coupled domains have 34 grid points in total. The computation of the rightmost lying eigenvalue in Figure 6 is resolved and the variation with $r$ is small. For a coarse mesh the convergence to steady-state can be slightly improved by having an unsymmetric coupling. This is again contrary to the result in [6].

\subsubsection{Error analysis}

We will use the method of manufactured solutions to study the error as a function of the interface parameter $r$. Any function $v \in C^{2}$ is a solution to

$$
\begin{aligned}
u_{t} & =u_{x x}+F(x, t), \quad-1 \leq x \leq 1, \\
u(x, 0) & =v(x, 0) \\
u(-1, t) & =v(-1, t) \\
u(1, t) & =v(1, t)
\end{aligned}
$$

where the forcing function $F(x, t)$ has been chosen appropriately. In this particular case we choose

$$
v(x, t)=\frac{\sin (2 \pi x-t)+\sin (t)}{4}
$$

which satisfies (49) with homogeneous boundary conditions and

$$
F(x, t)=\frac{\cos (t)-\cos (2 \pi x-t)+\pi^{2} \sin (2 \pi x-t)}{4} .
$$

The spatial discretization and stability conditions are the same as before and we use the classical 4th-order Runge-Kutta time integration scheme to solve a system of the form

$$
\frac{\partial \psi}{\partial t}=M \psi+F
$$


All spatial discretization, including boundary and interface conditions, is included in $M$ and $F$ is the above forcing function in discrete vector form. Thus we have a analytical solution which we can use to study the errors. In [17] it is stated that the errors can be reduced depending on the interface coupling for a hyperbolic problem and we will investigate if a similar effect exist for a parabolic problem.

Using the manufactured solution (49) it was verified that the coupled scheme converges with second order of accuracy independent of the interface parameter $r$.

In Figure 7 we plot the $l_{\infty}$-difference between the uncoupled and coupled equations for $r=-1,0$ and $r=-\frac{1}{2}$. Note that this is not a comparison with the analytic solution. We can see that there is no difference between the coupled and uncoupled solution for $r=-\frac{1}{2}$. In Figure 8 the difference between the analytic and computed

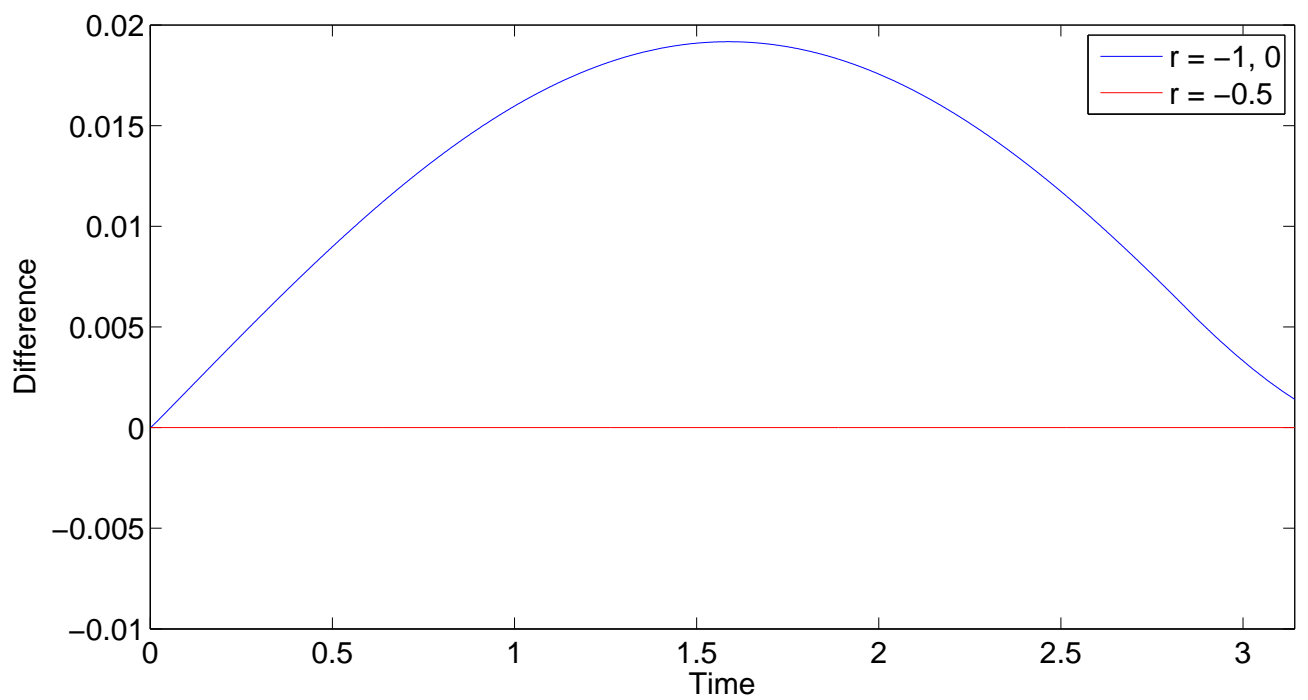

Figure 7: $l_{\infty}$-difference in time between the coupled and uncoupled solutions for different values of $r$ with 33 grid points for the single domain and 34 for the multi domain.

solution is plotted for $r=-1,0$ and $r=-\frac{1}{2}$ at time $t=\frac{\pi}{2}$. This time was chosen since the manufactured solution is $\pi$-periodic and the largest errors occur at $t=\frac{\pi}{2}$. We can see that for $r=-1,0$ the errors are smaller than for $r=-\frac{1}{2}$ in one domain but larger in the other. The total $l_{2}$-error is shown in Table 1 where we can see that the global error is slightly smaller when using a symmetric coupling.

Table 1: $l_{2}$-error for 34 grid points and different values of $r$

\begin{tabular}{|c|c|c|c|}
\hline$r$ & -1 & 0 & $-1 / 2$ \\
\hline$l_{2}$-error & 0.0057 & 0.0057 & 0.0055 \\
\hline
\end{tabular}

In Figure 9 we plot the $l_{\infty}$-error as a function of $r$. We can see that the maximum error can be reduced by almost 35 percent by chosing $r$ such that the equations are coupled symmetrically. 


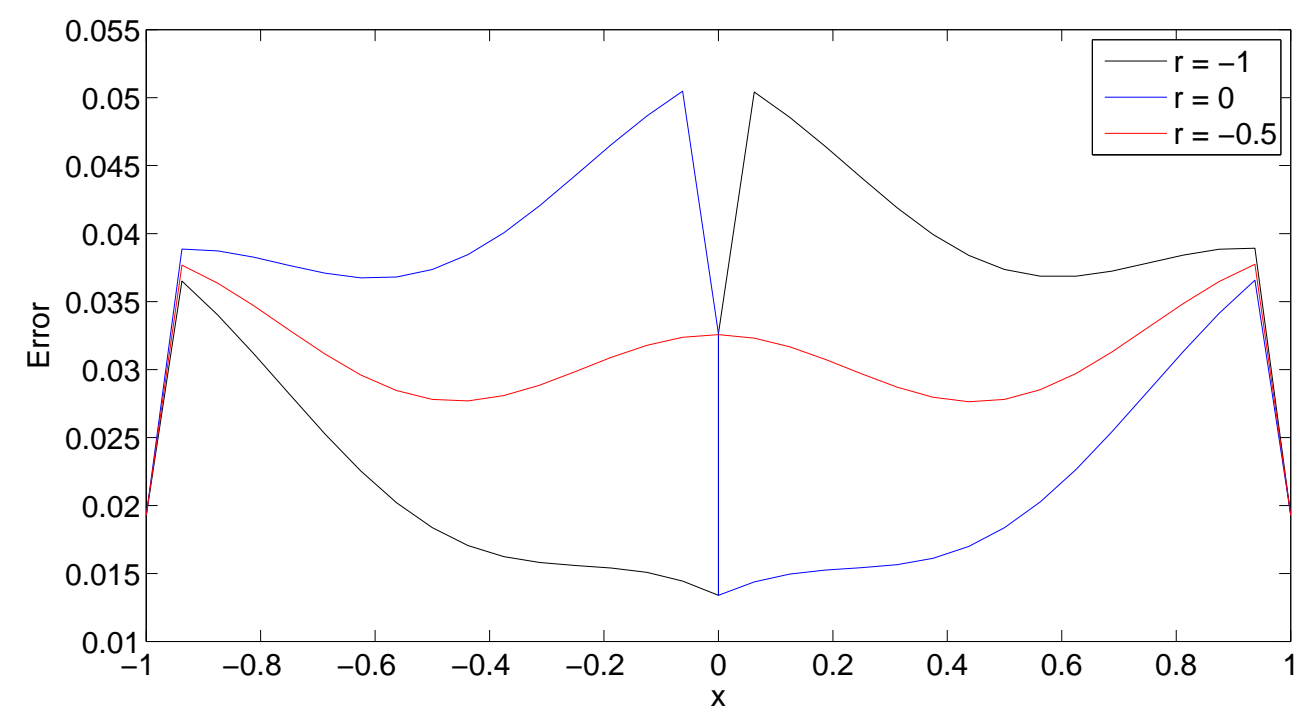

Figure 8: Difference at $t=\frac{\pi}{2}$ between the analytic and computed solution with 34 grid points for different values of $r$

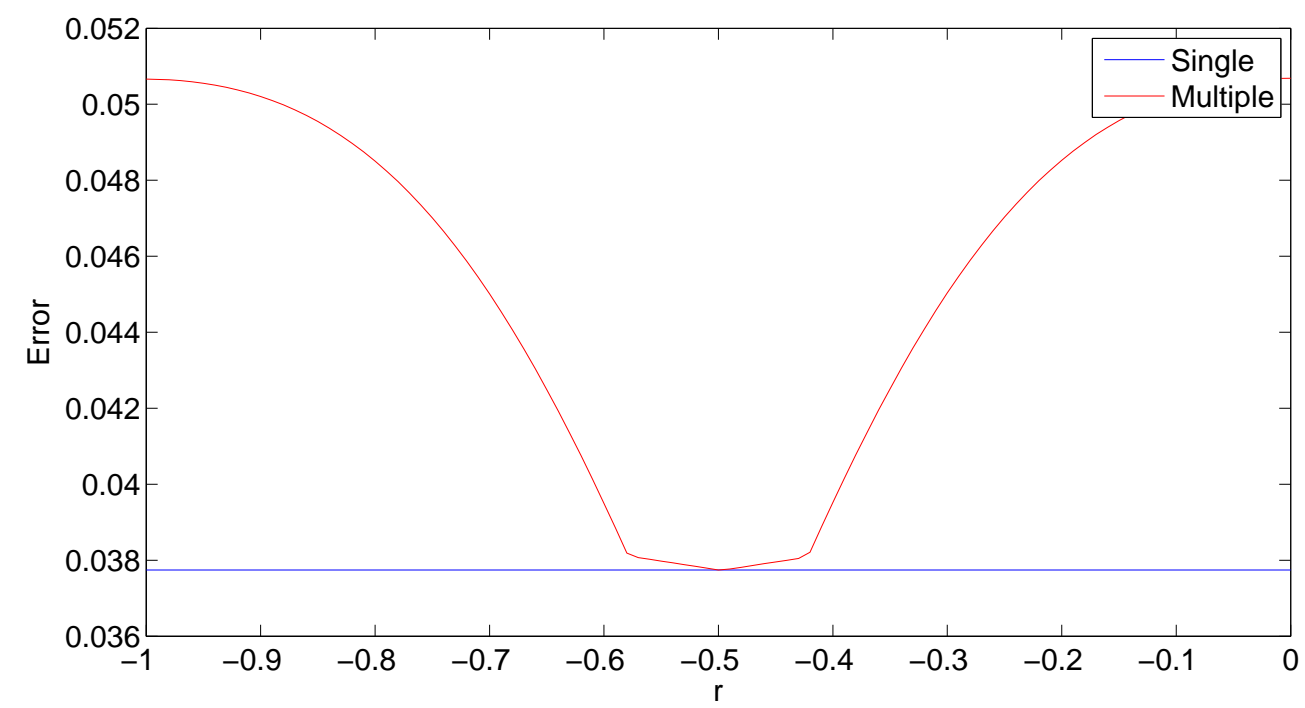

Figure 9: $l_{\infty}$-error at $t=\frac{\pi}{2}$ as a function of $r$ with 33 grid points for the single domain and 34 for the multi domain.

\section{Single domain spectral analysis of the advection equation}

We shall perform an analogous analysis for the advection equation as for the heat equation to see if similar results hold for the advection operator. 


\subsection{Continuous case}

Consider the advection equation in one domain,

$$
\begin{aligned}
u_{t}+u_{x} & =0, \quad-1 \leq x \leq 1, \\
u(-1, t) & =g(t), \\
u(x, 0) & =f(x) .
\end{aligned}
$$

Equation (53) is significantly different from (1) due to the directionality of the spatial operator. In this case there is one signal travelling from left to right and hence only one boundary condition is needed at $x=-1$. To obtain the spectrum we take the Laplace transform of (53) and proceed as before. We get

$$
s \hat{u}+\hat{u}_{x}=0
$$

which has the characteristic equation

$$
\kappa+s=0
$$

and thus the general solution of (54) is

$$
\hat{u}=c e^{-s x} .
$$

If we apply the boundary condition with $g=0$ we get $c=0$ and thus $\hat{u}=0$. Hence there is no continuous spectrum of (53) since there are no values of $s$ such that $c e^{s}=0$ for $c \neq 0$.

\subsection{Discrete case}

We discretize (53) using the SBP and SAT technique on a uniform mesh of $N+1$ grid points

$$
u_{t}+P^{-1} Q v=\sigma P^{-1}\left(v_{0}-g\right) e_{0}
$$

where $P$ and $e_{0}$ are as before and

$Q=\frac{1}{2}\left[\begin{array}{cccccc}-1 & 1 & 0 & 0 & \cdots & 0 \\ -1 & 0 & 1 & 0 & \cdots & 0 \\ \vdots & \ddots & \ddots & \ddots & \ddots & \vdots \\ 0 & \cdots & 0 & -1 & 0 & 1 \\ 0 & \cdots & 0 & 0 & -1 & 1\end{array}\right], P^{-1} Q=\frac{1}{2 \Delta x}\left[\begin{array}{cccccc}-2 & 2 & 0 & 0 & \cdots & 0 \\ -1 & 0 & 1 & 0 & \cdots & 0 \\ \vdots & \ddots & \ddots & \ddots & \ddots & \vdots \\ 0 & \cdots & 0 & 1 & 0 & -1 \\ 0 & \cdots & 0 & 0 & -2 & 2\end{array}\right]$.

Note that $Q+Q^{T}=\operatorname{diag}(-1,0, \cdots, 0,1)$ which is used to select the boundary terms in the energy estimate. By applying the energy method to (57) with $g=0$ we get

$$
\|v\|_{t}^{2}=(1+2 \sigma) v_{0}^{2}-v_{N}^{2}
$$

which is bounded for $\sigma \leq-\frac{1}{2}$ and hence the scheme is stable.

To determine the spectrum we Laplace transform (57) (with $g=0$ ) and rewrite as

$$
\left(s I+P^{-1} Q-\sigma P^{-1} E_{0}\right) \hat{v}=0 .
$$


From the internal scheme we have

$$
s \hat{v}_{i}+\frac{1}{2 \Delta x}\left(\hat{v}_{i+1}-\hat{v}_{i-1}\right)=0
$$

or equivalently

$$
\hat{v}_{i+1}+2 \tilde{s} \hat{v}_{i}-\hat{v}_{i-1}=0
$$

with $\tilde{s}=s \Delta x$. The characteristic equation is $\kappa^{2}+2 \tilde{s} \kappa-1=0$ which has solutions

$$
\kappa_{+,-}=-\tilde{s} \pm \sqrt{\tilde{s}^{2}+1} .
$$

Thus the general solution of (62) is

$$
\hat{v}_{i}=c_{1} \kappa_{+}^{i}+c_{2} \kappa_{-}^{i} .
$$

The first and last equation in (60) are modified and we can use them to write a matrix equation for the unknowns $c_{1,2}$. The equations are

$$
\begin{aligned}
& (\tilde{s}-1-2 \sigma) \hat{v}_{0}+\hat{v}_{1}=0 \\
& -v_{N-1}+(\tilde{s}+1) \hat{v}_{N}=0
\end{aligned}
$$

and by inserting the general solution (64) into (65) we get the matrix equation $E(s, \kappa) c=0$ where

$$
E(s, \kappa)=\left[\begin{array}{cc}
\tilde{s}-1-s \sigma+\kappa_{+} & \tilde{s}-1-s \sigma+\kappa_{-} \\
(\tilde{s}+1) \kappa_{+}^{N}-\kappa_{+}^{N-1} & (\tilde{s}+1) \kappa_{-}^{N}-\kappa_{-}^{N-1}
\end{array}\right] .
$$

The spectrum consists as before of the singular points of $E(s, \kappa)$. A direct computation of the determinant of $E(s, \kappa)$ gives that

$$
\begin{aligned}
\operatorname{det}(E(s, \kappa)) & =\kappa_{-}^{N}\left(\sqrt{\tilde{s}^{2}+1}-1-2 \sigma\right)\left(1+\sqrt{\tilde{s}^{2}+1}\right) \\
& +\kappa_{+}^{N}\left(\sqrt{\tilde{s}^{2}+1}+1+2 \sigma\right)\left(1-\sqrt{\tilde{s}^{2}+1}\right) .
\end{aligned}
$$

A closed form expression for the zeros of (67) have not been found. We can however compute the eigenvalues numerically. We will return to (67) when we consider the spectrum of the coupled problem.

\section{Multi domain spectral analysis of the advection equation}

We introduce again an artificial interface at $x=0$ for the advection equation to study how the spectral properties of the continuous and discrete operators are modified.

\subsection{Continuous case}

Consider now

$$
\begin{aligned}
u_{t}+u_{x} & =0, \quad-1 \leq x \leq 0, \\
v_{t}+v_{x} & =0, \quad 0 \leq x \leq 1, \\
u(-1, t) & =g(t) \\
v(0, t) & =u(0, t) .
\end{aligned}
$$


The spectrum is again obtained by Laplace transforming (68) and applying the boundary and interface conditions. The general solutions to the Laplace transformed equations are $\hat{u}=c_{1} e^{-s x}$ and $\hat{v}=c_{2} e^{-s x}$. The boundary and interface conditions imply that $c_{1}=0$ and $c_{2}=c_{1}$, and hence there is no spectrum as expected.

\subsection{Discrete case}

One form of the SBP and SAT discretization of (68) is

$$
\begin{aligned}
u_{t}+P^{-1} Q u & =\sigma P^{-1}\left(u_{0}-g\right) e_{0} \\
v_{t}+P^{-1} Q v & =\tau P^{-1}\left(v_{0}-u_{N}\right) e_{0}
\end{aligned}
$$

where $u, v$ now denote the discrete grid functions. Both domains have equidistant grid spacing and equal number of grid points to allow for the same difference operators in both domains.

\subsubsection{Conservation and stability}

When constructing an interface for equations with advection it is important that the scheme is not only stable, but also conservative $[1,18,10]$. Let $\Phi(x)$ be a smooth testfunction and let $\phi=\left[\Phi\left(x_{0}\right), \ldots, \Phi\left(x_{N}\right)\right]^{T}$. We multiply both equations in (69) with $\phi^{T} P$ respectively. By using the SBP property of $Q$ and adding the two equations we can shift the differentiation onto $\phi$ and get

$$
\phi^{T} P u_{t}+\phi^{T} P v_{t}-\phi_{0} u_{0}+\phi_{N} v_{N}-(Q \phi)^{T} u-(Q \phi)^{T} v-\sigma \phi_{0}\left(u_{0}-g\right)=\phi_{i}(\tau+1)\left(v_{0}-u_{N}\right)
$$

where we have used $\phi_{0}=\Phi(-1), \phi_{N}=\Phi(1)$ and $\phi_{i}=\Phi(0)$ to denote the boundary and interface points. To ensure that the interface is conservative we cannot have any remaining interface terms and hence we need to put $\tau=-1$ to cancel the terms in the right hand side of (70). With this choice we thus have a conservative interface treatment.

To determine the stability condition we proceed with the energy method as before by multiplying both equations in (69) with $u^{T} P$ and $v^{T} P$ respectively. By assuming that $g=0$ we get

$$
\|u\|_{t}^{2}+\|v\|_{t}^{2}=(1+2 \sigma) u_{0}^{2}+(1+\tau) v_{0}^{2}-v_{N}^{2}-\left(u_{N}+\tau v_{0}\right)^{2}
$$

and we can see that the scheme is stable if we chose $\sigma \leq-\frac{1}{2}$ and $\tau \leq-1$. Thus the interface treatment is both stable and conservative with $\tau=-1$.

\subsubsection{Eigenspectrum}

We Laplace transform (69) and get the general solution from the internal schemes as before,

$$
\begin{aligned}
& \hat{u}_{i}=c_{1} \kappa_{+}^{i}+c_{2} \kappa_{-}^{i} \\
& \hat{v}_{i}=c_{3} \kappa_{+}^{i}+c_{4} \kappa_{-}^{i},
\end{aligned}
$$


where $\kappa_{+,-}=-\tilde{s} \pm \sqrt{\tilde{s}^{2}+1}$. The scheme at the boundaries and interfaces are different from the internal scheme and their corresponding equations are

$$
\begin{aligned}
(\tilde{s}-1-2 \sigma) \hat{u}_{0}+\hat{u}_{1} & =0 \\
(\tilde{s}+1) \hat{u}_{N}-\hat{u}_{N-1} & =0 \\
2 \tau \hat{u}_{N}+(\tilde{s}-1-2 \tau) \hat{v}_{0}+\hat{v}_{1} & =0 \\
(\tilde{s}+1) \hat{v}_{N}-\hat{v}_{N-1} & =0 .
\end{aligned}
$$

By inserting the general solutions into (73) we get again the matrix equation $E(s, \kappa) c=$ 0 for the unknowns $c=\left[c_{1}, \ldots, c_{4}\right]^{T}$ where

$$
E(s, \kappa)=\left[\begin{array}{cccc}
\tilde{s}-1-2 \sigma+\kappa_{+} & \tilde{s}-1-2 \sigma+\kappa_{-} & 0 & 0 \\
(\tilde{s}+1) \kappa_{+}^{N}-\kappa_{+}^{N-1} & (\tilde{s}+1) \kappa_{-}^{N}-\kappa_{-}^{N-1} & 0 & 0 \\
2 \tau \kappa_{+}^{N} & 2 \tau \kappa_{-}^{N} & \tilde{s}-1-2 \tau+\kappa_{+} & \tilde{s}-1-2 \tau+\kappa_{-} \\
0 & 0 & (\tilde{s}+1) \kappa_{+}^{N}-\kappa_{+}^{N-1} & (\tilde{s}+1) \kappa_{-}^{N}-\kappa_{-}^{N-1}
\end{array}\right] .
$$

The spectrum is obtained for the singular values of $E(s, \kappa)$. By expanding the determinant of $E(s, \kappa)$ and factorizing we get

$$
\operatorname{det}(E(s, \kappa))=f(\sigma, s) g(\tau, s)
$$

where

$$
\begin{aligned}
f(\sigma, s) & =\kappa_{-}^{N}\left(\sqrt{\tilde{s}^{2}+1}-1-2 \sigma\right)\left(1+\sqrt{\tilde{s}^{2}+1}\right) \\
& +\kappa_{+}^{N}\left(\sqrt{\tilde{s}^{2}+1}+1+2 \sigma\right)\left(1-\sqrt{\tilde{s}^{2}+1}\right)
\end{aligned}
$$

is exactly (67). The second factor is

$$
\begin{aligned}
g(\tau, s) & =\left(\tilde{s}^{2}-1-2 \tau \tilde{s}-2 \tau+\tilde{s} \kappa_{+}+\kappa_{+}\right) \kappa_{-}^{N} \\
& -\left(\tilde{s}^{2}-1-2 \tau \tilde{s}-2 \tau+\tilde{s} \kappa_{-}+\kappa_{-}\right) \kappa_{+}^{N} \\
& -\left(\tilde{s}-1-2 \tau+\kappa_{+}\right) \kappa_{-}^{N-1}+\left(\tilde{s}-1-2 \tau+\kappa_{-}\right) \kappa^{N-1} .
\end{aligned}
$$

We can see that the single domain operator spectrum is again contained in the multi domain operator spectrum, which is visualized in Figure 10 and Figure 11 for $\sigma=-\frac{1}{2}$ and $\sigma=-1$ respectively. In the second case, which is fully upwinded, we can see that the spectrum is identical for the single and multi domain operators since all eigenvalues of the multi domain operator are double eigenvalues.

Figure 12 shows the spectrum of both the single and multi domain operators for 33 and 34 grid points respectively and $\sigma=-1$. We can see that the spectrum of the operators does not agree as they did in the coupled diffusion case. Note again that all eigenvalues of the multi domain operator are double eigenvalues.

\subsection{Extending the interface treatment}

In the previous section we discussed one of many different schemes for the advection equation coupled over an interface. The scheme was based on the boundary and interface conditions for the continuous PDE. The interface condition $v=u$ is of 


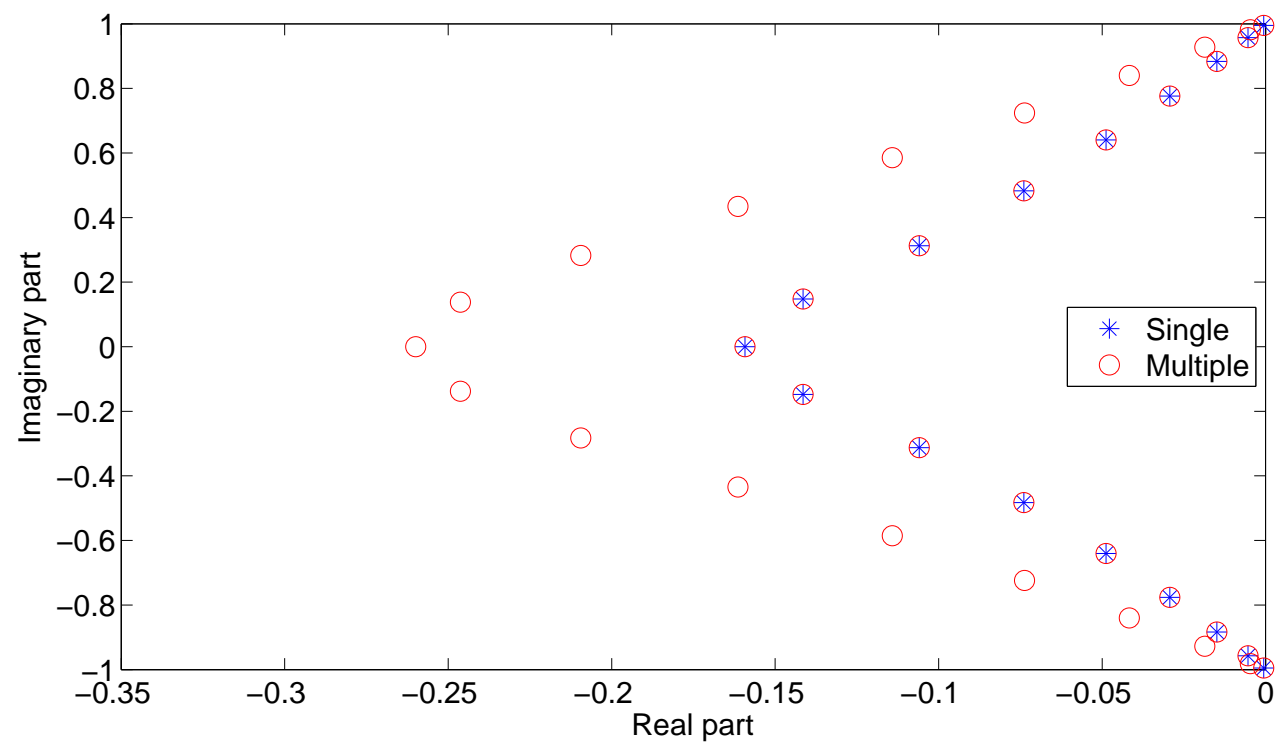

Figure 10: Spectrum of both the single and multi domain operator with $\sigma=-\frac{1}{2}$ and scaled with $\Delta x$, using 17 grid points for the single domain operator and 34 for the multi domain operator. The single domain operator spectrum is contained in the multi domain operator spectrum.

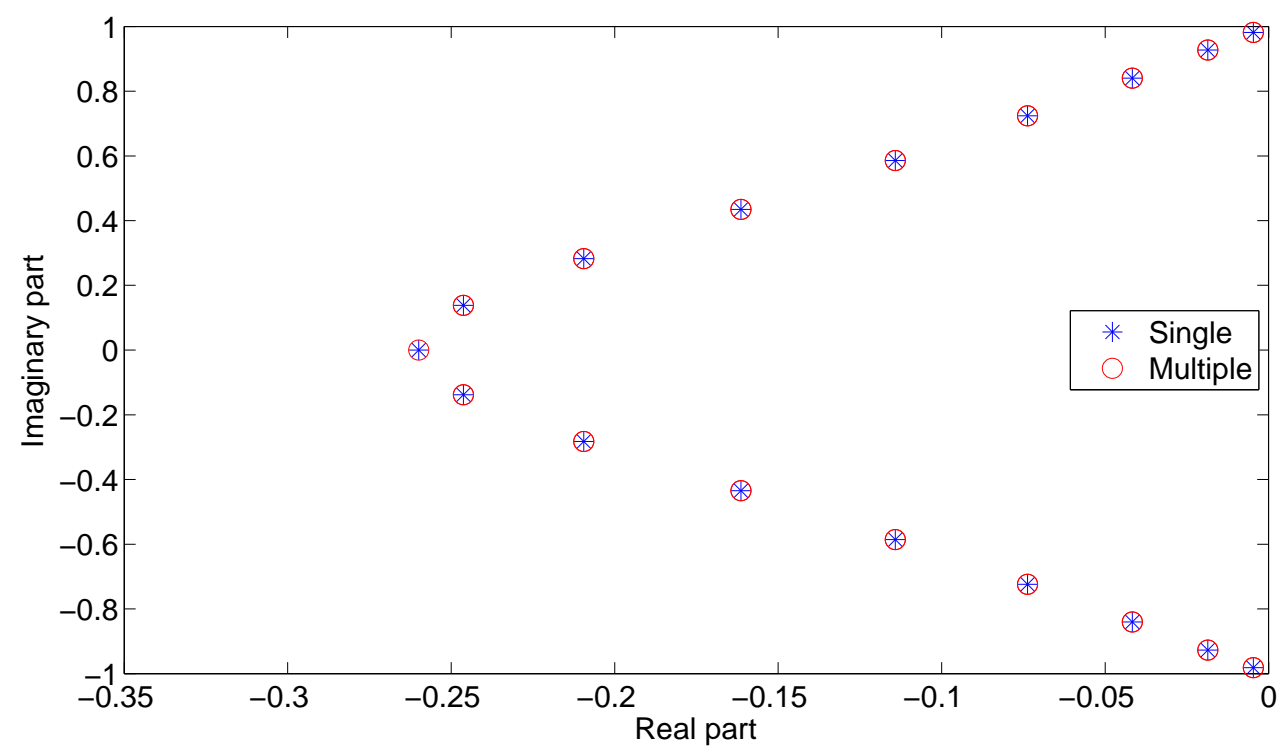

Figure 11: Spectrum of both the single and multi domain operator with $\sigma=-1$ and scaled with $\Delta x$, using 17 grid points for the single domain operator and 34 for the multi domain operator. The multi domain operator spectrum is identical to the single domain operator spectrum since all eigenvalues of the multi domain operator are double eigenvalues.

course identical to $u=v$ in the continuous sense, but this is not true in the discrete setting with weak interface conditions. We can hence modify the interface treatment 


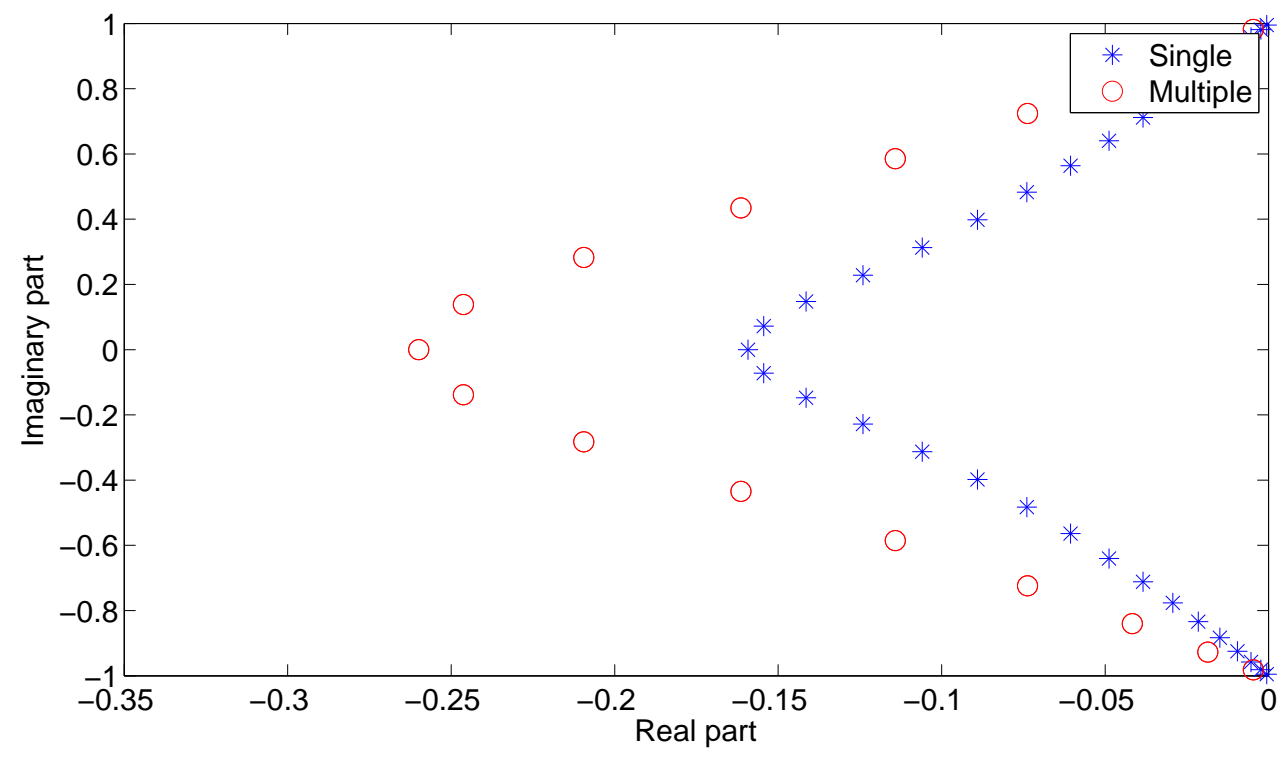

Figure 12: Spectrum of both the single and multi domain operator with $\sigma=-1$ and scaled with $\Delta x$ using 33 grid points for the single domain operator and 34 for the multi domain operator

by adding one additional term corresponding to $u=v$ in the discrete setting.

Consider the scheme (69) again but without the outer boundary term and with one additional term added,

$$
\begin{aligned}
u_{t}+P^{-1} Q u & =\gamma P^{-1}\left(u_{N}-v_{0}\right) e_{N} \\
v_{t}+P^{-1} Q v & =\tau P^{-1}\left(v_{0}-u_{N}\right) e_{0} .
\end{aligned}
$$

The stability and conservation criteria can be found in e.g. [19] so we just state the result here as a proposition,

Proposition 5.1. The interface scheme (78) is stable and conservative for

$$
\begin{aligned}
\gamma & =\frac{1-\theta}{2} \\
\tau & =-\frac{1+\theta}{2}
\end{aligned}
$$

where $\theta \geq 0$ is a free parameter.

The energy estimate of (78) is

$$
\|u\|_{t}^{2}+\|v\|_{t}^{2}=-\theta\left(u_{N}^{2}-v_{0}^{2}\right)
$$

when ignoring the outer boundary terms. Note that $\theta=1$ gives (69) which is fully upwinded while $\theta=0$ gives minimal dissipation. By Taylor expanding (78) it can easily be seen that the formal accuracy is independent of the choice of $\theta$. We did a convergence study and verified that the solution converges with second order accuracy independently of the choice of parameters. 


\subsubsection{Stiffness, convergence and errors}

In the case of advection there are two free parameters compared to the diffusion case where there is only one. One parameter for the outer boundary $-\infty \leq \sigma \leq-\frac{1}{2}$ and one parameter for the interface $0 \leq \theta \leq \infty$. Since we are interested only in the interface treatment we let $\sigma=-1$ be fixed and consider the stiffness, rate of convergence and error as a function of $\theta$.

In [17] the quasi-one-dimensional Euler equations were used with an interface treatment corresponding to $\theta=1$ to study the errors. Their convergence study showed that the errors were small and does not increase with the number of subdomains. We continue with a more detailed investigation by posing the errors as functions of the interface treatment.

We use the manufactured solution $u(x, t)=\sin (2 \pi(x-t))$ to study the errors as a function of $\theta$. Using this solution we construct initial and boundary data to use in the error analysis. The maximum error is shown in Figure 13. We can see that for $\theta \geq 1$, the maximum error drops to the same level as for the single domain problem. Compared to the minimal dissipative case, the maximum error is approximately 20 percent smaller.

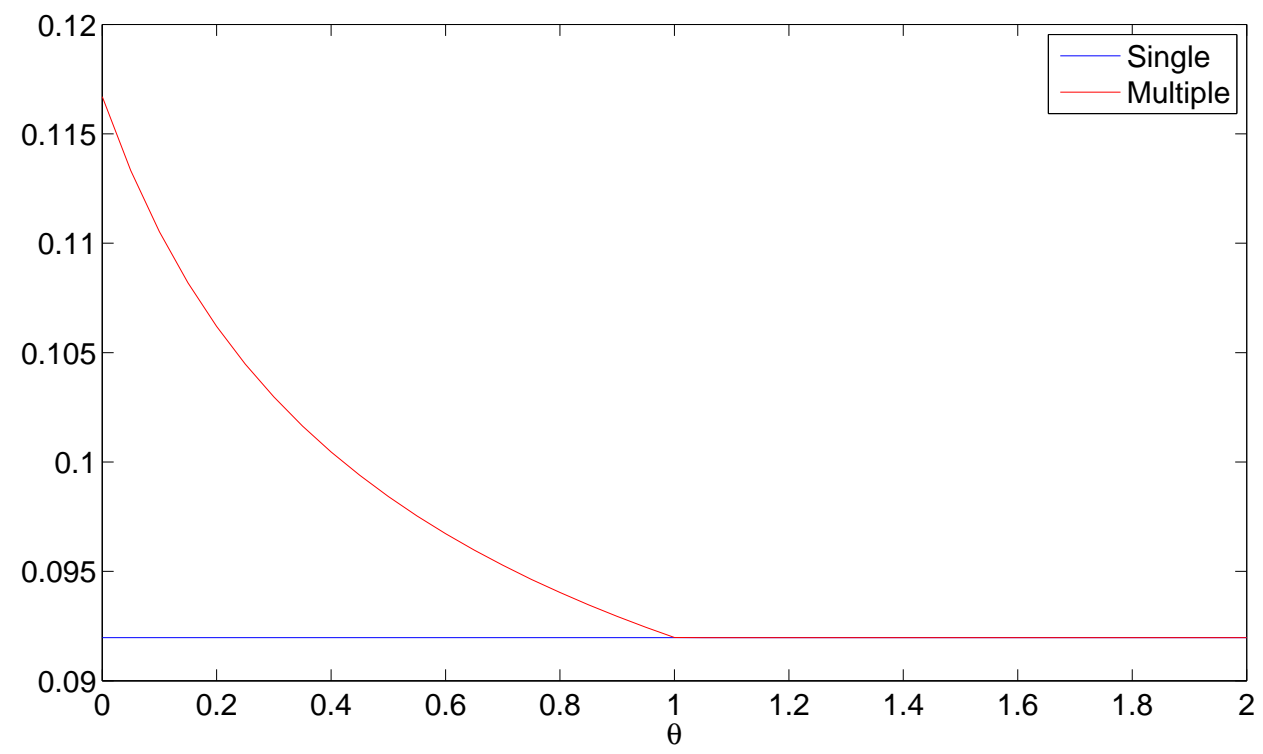

Figure 13: $l_{\infty}$-error of the single and multi domain operator as a function of $\theta$ using 33 and 34 grid points respectively.

The stiffness and rate of convergence are shown in Figures (14), (15) where 33 grid points are used for the single domain and 34 for the multi domain. We can see from Figures (14) and (15) that it is possible to maintain and even improve the stiffness and rate of convergence when $\theta=1$ which is the fully upwinded scheme.

The rate of convergence is improved by approximately a factor seven when the interface is fully upwinded. We can visualize the effect by performing a computation to steady-state and measure the decay rate. We consider a Gaussian disturbance as 


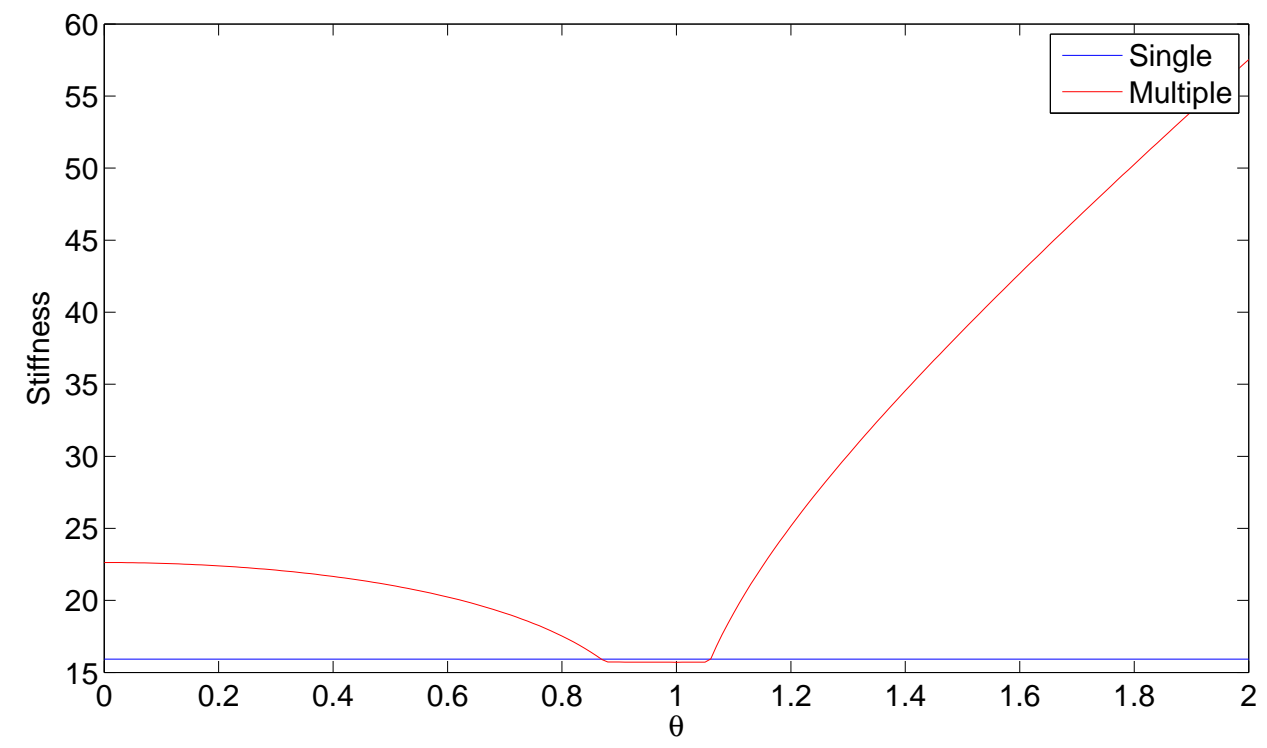

Figure 14: $\max (\operatorname{abs}(\lambda))$ as function of $\theta$. The single domain operator is using 33 grid points and the multi domain operator is using 34 grid points.

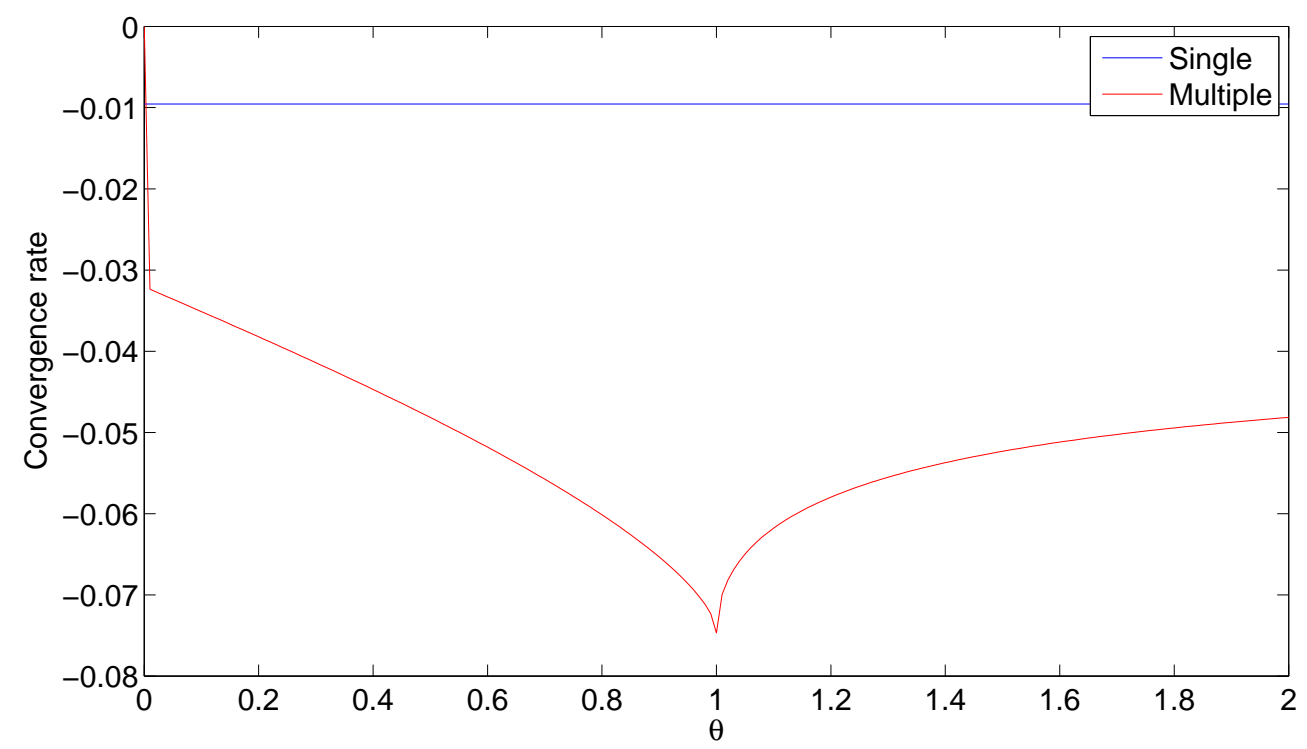

Figure 15: $\max (\mathbb{R}(\lambda))$ as a function of $\theta$. The single domain operator is using 33 grid points and the multi domain operator is using 34 grid points.

initial data with initial position $x_{0}=-\frac{1}{2}$. The initial data is given explicitly by

$$
f(x)=e^{-100\left(x-x_{0}\right)^{2}} .
$$

The disturbance is transported out of the boundary and the exact steady-state solution is identically zero. To investigate what effect multiple interfaces have we compute in time until the $l_{2}$-norm of the solution is less than $10^{-16}$, which is considered to be the steady-state solution. The result is seen in Table 2 for the single 
Table 2: The time at which the $l_{2}$-norm of the solution is less than $10^{-16}$ for $1,2,4$ and 8 domains.

\begin{tabular}{|c|c|c|c|c|}
\hline \# domains & 1 & 2 & 4 & 8 \\
\hline$\theta=1$ & 6021.6 & 912.0 & 146.5 & 28.8 \\
\hline
\end{tabular}

domain case and the multi domain case with 2, 4 and 8 domains. The number of grid points in the single domain case is 48 and is increased by 1 for each additional domain. We can see that there is a huge increase in gain to reach steady-state when more upwinded interfaces are introduced. Note that the time to reach steady-state for one and two domains differ by almost a factor seven which is what we can expect from Figure 15. The spectra of all cases for $\theta=1$ is seen in Figure 17. We can

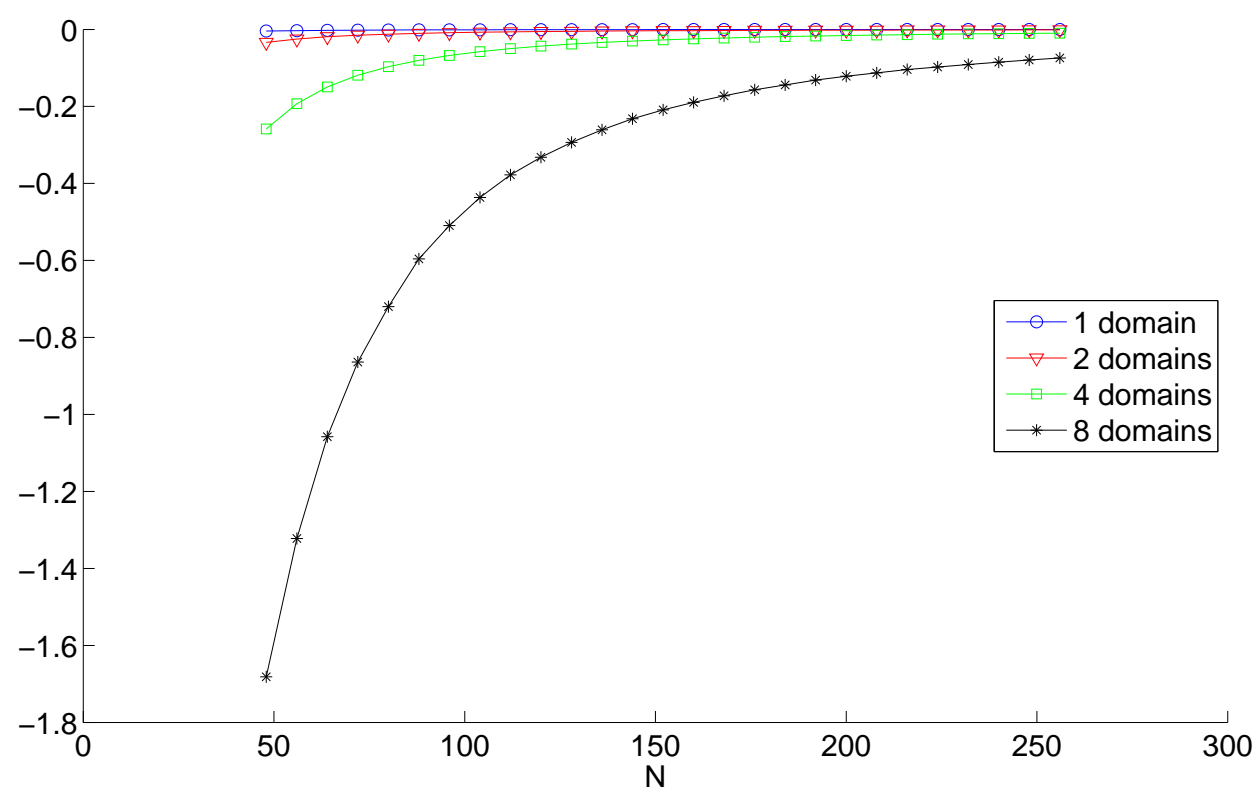

Figure 16: $\max (\mathbb{R}(\lambda))$ as a function of $N$ with $\theta=1$.

see that the real part of the eigenvalues are shifted further to the left when more upwinded interfaces area added.

To have minimally dissipative interfaces greatly reduces the rate of convergence to far below the level of the single domain case. We have performed steady-state computations using $\theta=0$ and verified that the time to reach steady-state is an order of magnitude slower than for the single domain case. This fact can also be seen from Figure 15 where there is an eigenvalue with real part very close to zero for $\theta=0$.

Remark 5.1. Independently of the number of interfaces we can pose the complete scheme as $\tilde{P} w_{t}=\tilde{Q} w+F$ where $\tilde{P}$ is the norm and all differentiation is collected in $\tilde{Q}$. The minimally dissipative interface treatment with $\theta=0$ renders $\tilde{Q}$ completely skew-symmetric except at the boundary points. 


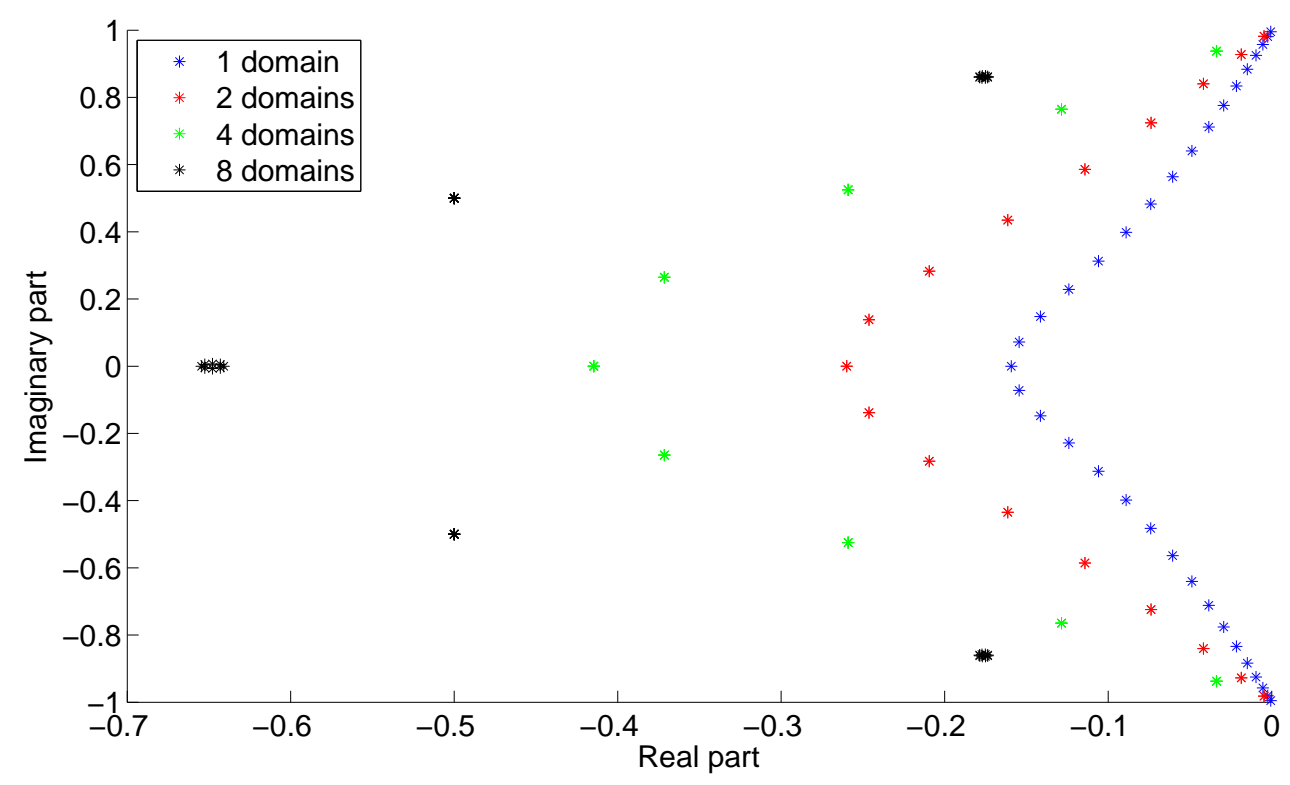

Figure 17: Spectra of the 1-, 2-, 4- and 8-domain operator scaled with $\Delta x$ for $\theta=1$. Note that the eigenvalues of the 8-domain operator are clustered.

\section{Verification of results for high order accurate approximations}

In the previous sections the derived results were for second order accurate operators. The choice of second order was made since it was possible to derive analytical results. In this section we discuss the results for a 4th order accurate finite SBP operator. Details on the operators can be found in $[7,8]$.

\subsection{Diffusion}

The stability conditions are independent of the order of accuracy. We can thus replace the difference operators in the scheme with their 4th order accurate correspondance. The analogue of Figure 2 is shown in Figure 18 where we can see that the single domain operator spectrum is contained in the multi domain operator spectrum. This is independent of the interface parameter $r$ and in Figure 18 we have chosen $r=-\frac{1}{2}$.

\subsection{Advection}

The stability and conservation criteria is independent of the order of accuracy. The scheme for the advection equation is thus identical except that the difference operator has been replaced by a 4th order accurate operator. The analogue of Figures 10 and 11 are shown in Figures 19 and 20. We can see that the single domain operator spectrum is contained in the multi domain operator spectrum. In the case of fully upwinded boundary and interface conditions, all eigenvalues of the multi domain operator are double eigenvalues and the spectra become identical. 


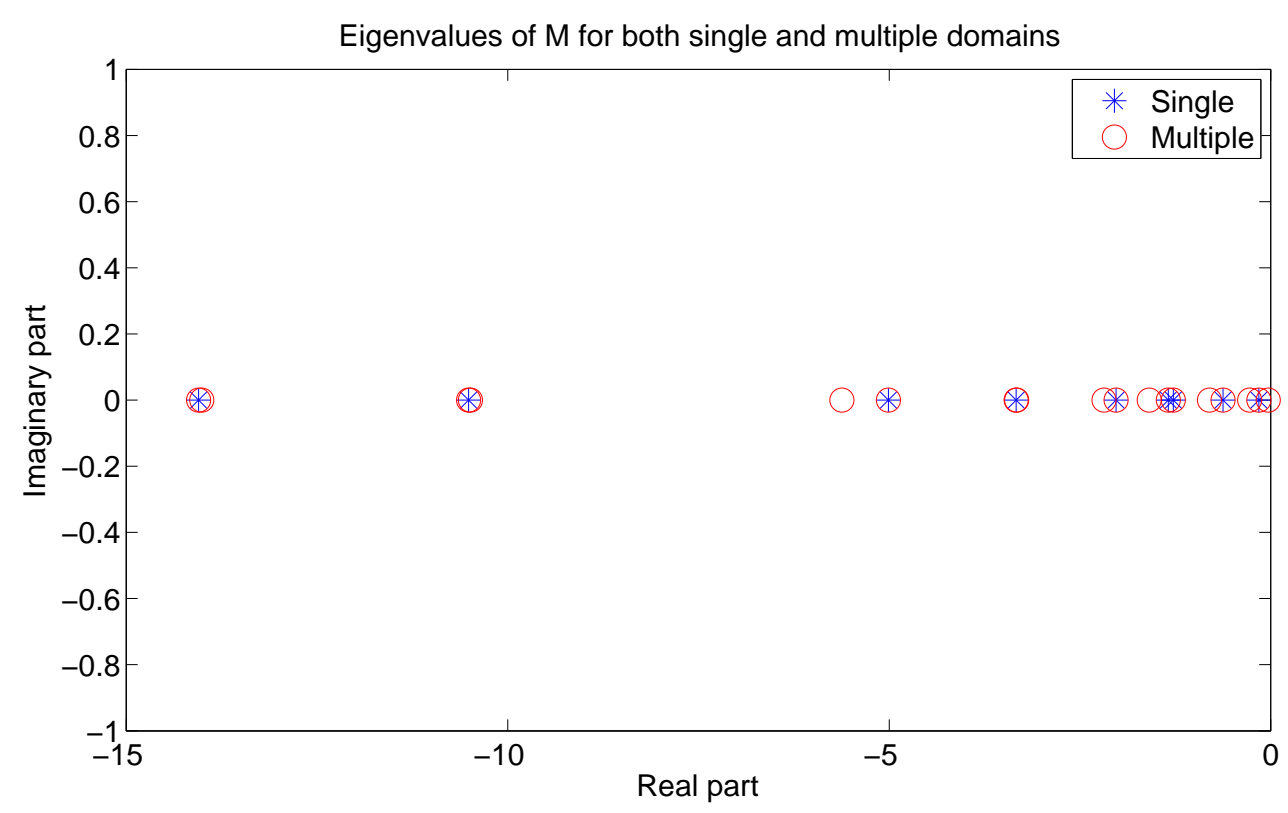

Figure 18: Eigenvalues of the 4th order accurate single domain diffusion operator with 9 grid points and the multi domain operator with 18 grid points scaled with $\Delta x^{2}$. The single domain operator spectrum is always contained in the multi domain operator spectrum independent of the interface parameter $r$. Here $r=-\frac{1}{2}$.

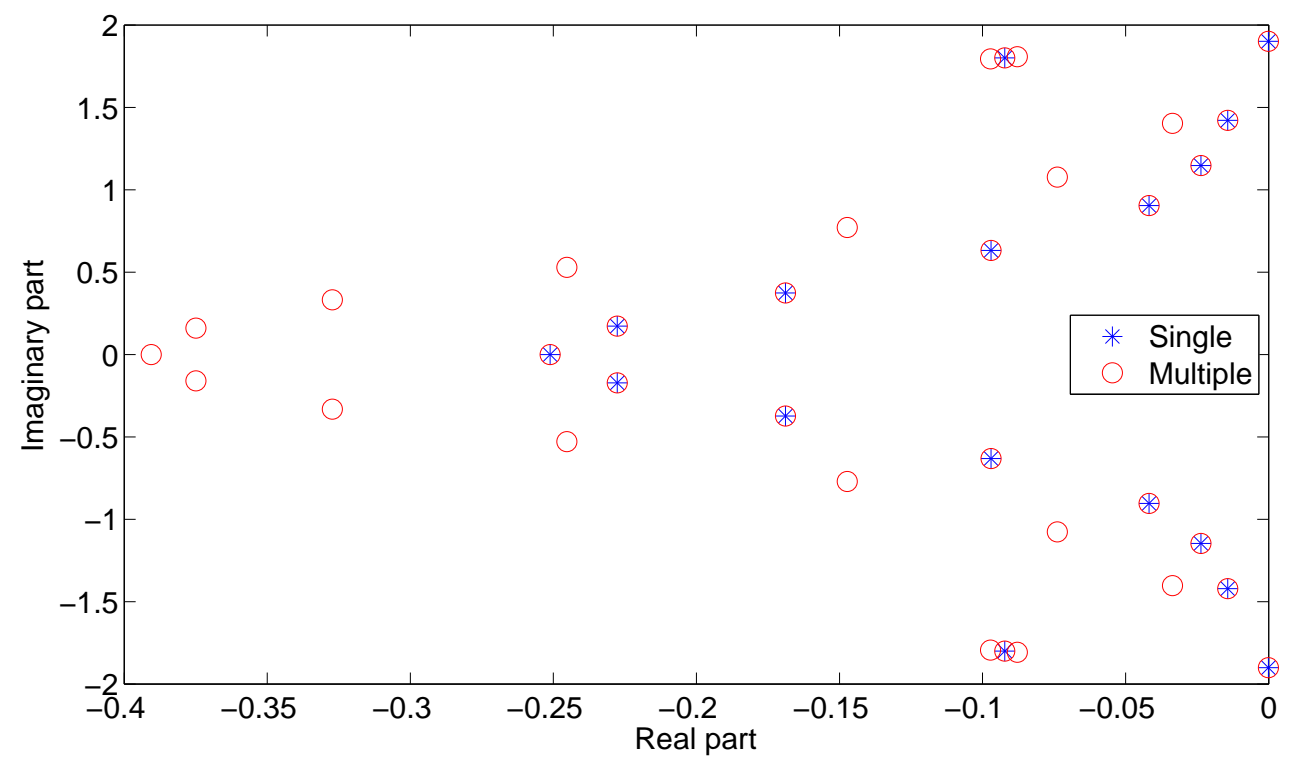

Figure 19: Spectrum of both the single and multi domain advection operator with $\sigma=-\frac{1}{2}$ and scaled with $\Delta x$, using 17 grid points for the 4th order accurate single domain operator and 34 for the multi domain operator. The single domain operator spectrum is contained in the multi domain operator spectrum.

\section{Summary and conclusions}

We have used a second order accurate finite difference method on Summation-ByParts form to discretize the heat- and advection equation on single and multiple 


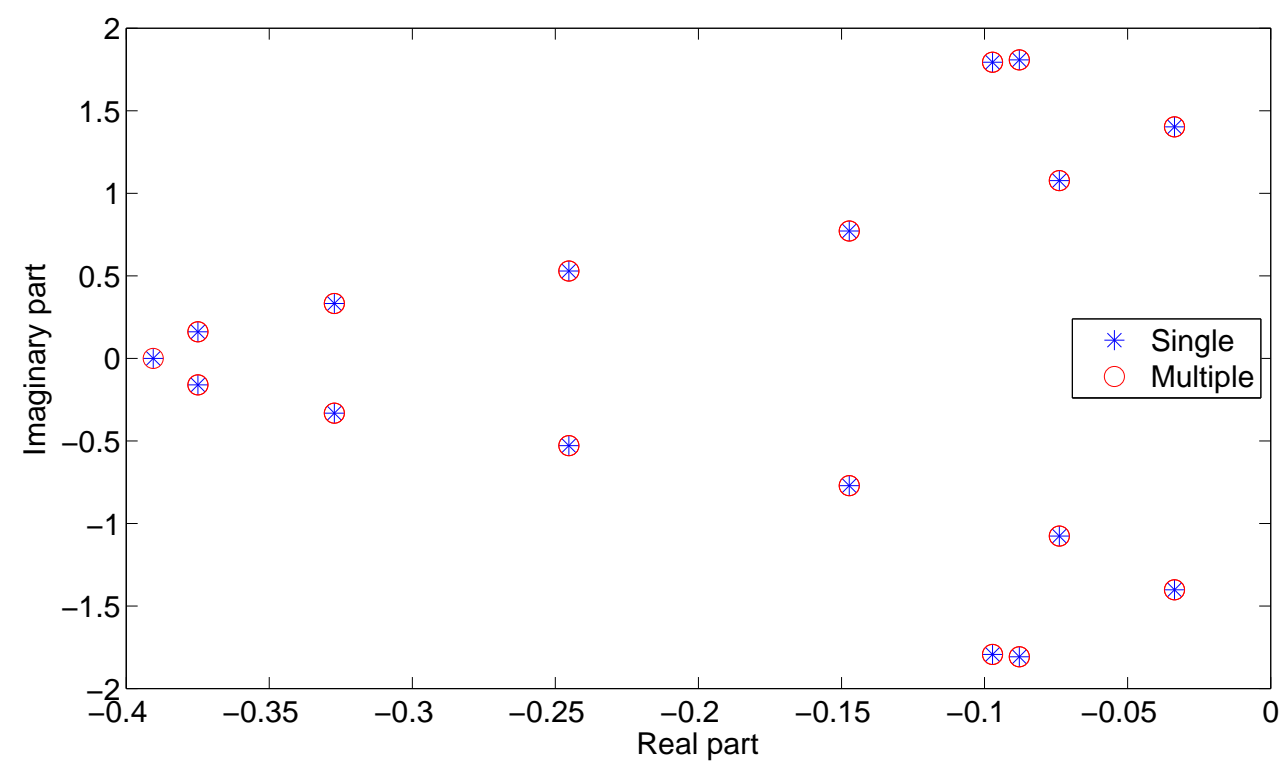

Figure 20: Spectrum of both the single and multi domain advection operator with $\sigma=-1$ and scaled with $\Delta x$, using 17 grid points for the 4th order accurate single domain operator and 34 for the multi domain operator. The multi domain operator spectrum is identical to the single domain operator spectrum since all eigenvalues of the multi domain operator are double eigenvalues.

domains. The results are differing between the diffusion and advection case and we discuss them separately.

In both cases we derived an interface treatment which is depending on an interface parameter which can be used to alter the interface treatment. We studied which impact the interface treatment has on the spectrum, stiffness, rate of convergence and errors. In both cases we showed how the spectrum of the single domain operator is included in the multi domain operator spectrum and that the result carry over to higher order accurate approximations.

\subsection{Diffusion}

In the single domain case, a closed form expression for all eigenvalues of the discretization matrix, including the boundary conditions, was found. We showed how the eigenvalues of the discretization matrix converged to the eigenvalues of the continuous equation. For the multiple domain case we showed how the spectrum of the single domain operator is contained in the multi domain operator spectrum independent of the interface treatment.

The stiffness and rate of convergence were not significantly effected by the choice of interface treatment. We used a manufactured solution to study the errors. When the symmetric coupling was used, the maximum error of the multi domain case reduced to the level of the single domain case. Compared to the unsymmetric coupling, the maximum errors were reduced by almost 35 percent when the symmetric coupling was used. 


\subsection{Advection}

For the advection equation we showed that the spectrum of the single domain operator is contained in the multi domain operator spectrum independent of the interface treatment similarly to the diffusion case.

The stiffness showed only minor differences depending on the interface treatment. The rate of convergence to steady-state was improved by approximately a factor seven when adding one upwinded interface. By adding more upwinded interfaces we could dramatically decrease the computational time to reach the steady-state solution. When the interface treatment was chosen minimally dissipative the scheme becomes completely skew-symmetric and the rate of convergence to steady-state was severely decreased due to the presence of an eigenvalue with almost zero real part.

We used an exact solution to study the errors as a function of the interface treatment. We showed that it is possible to bring down the maximum errors to the level of the single domain case by using the upwinded coupling. The maximum error was about 20 percent smaller when using a fully upwinded coupling compared to the minimal dissipative coupling.

\section{References}

[1] Jan Nordström, Jing Gong, Edwin van der Weide, and Magnus Svärd. A stable and conservative high order multi-block method for the compressible NavierStokes equations. Journal of Computational Physics, 228(24):9020-9035, 2009.

[2] Jan Nordström and Magnus Svärd. Well-Posed Boundary Conditions for the Navier-Stokes Equations. SIAM Journal on Numerical Analysis, 43(3):1231$1255,2005$.

[3] Magnus Svärd, Mark H. Carpenter, and Jan Nordström. A stable high-order finite difference scheme for the compressible Navier-Stokes equations, far-field boundary conditions. Journal of Computational Physics, 225(1):1020-1038, 2007.

[4] Magnus Svärd and Jan Nordström. A stable high-order finite difference scheme for the compressible Navier-Stokes equations: No-slip wall boundary conditions. Journal of Computational Physics, 227(10):4805 - 4824, 2008.

[5] Magnus Svärd, Ken Mattsson, and Jan Nordström. Steady-State Computations Using Summation-by-Parts Operators. Journal of Scientific Computing, 24(1):79-95, 2005.

[6] Jens Lindström and Jan Nordström. A stable and high-order accurate conjugate heat transfer problem. Journal of Computational Physics, 229(14):5440-5456, 2010 .

[7] Ken Mattsson and Jan Nordström. Summation by parts operators for finite difference approximations of second derivatives. Journal of Computational Physics, 199(2):503-540, 2004. 
[8] Bo Strand. Summation by Parts for Finite Difference Approximations for d/dx. Journal of Computational Physics, 110(1):47 - 67, 1994.

[9] Bertil Gustafsson, Heinz-Otto Kreiss, and Joseph Oliger. Time Dependent Problems and Difference Methods. Wiley Interscience, 1995.

[10] Mark H. Carpenter, Jan Nordström, and David Gottlieb. Revisiting and extending interface penalties for multi-domain summation-by-parts operators. Journal of Scientific Computing, 45(1-3):118-150, 2010.

[11] Sofia Eriksson and Jan Nordström. Analysis of the order of accuracy for nodecentered finite volume schemes. Applied Numerical Mathematics, 59(10):2659 - 2676, 2009.

[12] Magnus Svärd and Jan Nordström. On the order of accuracy for difference approximations of initial-boundary value problems. Journal of Computational Physics, 218(1):333-352, 2006.

[13] Ken Mattsson. Boundary Procedures for Summation-by-Parts Operators. Journal of Scientific Computing, 18(1):133-153, 2003.

[14] Björn Engquist and Bertil Gustafsson. Steady state computations for wave propagation problems. Mathematics of Computation, 49(179):39-64, 1987.

[15] Jan Nordström. The influence of open boundary conditions on the convergence to steady state for the Navier-Stokes equations. Journal of Computational Physics, 85(1):210 - 244, 1989.

[16] Peter Eliasson, Sofia Eriksson, and Jan Nordström. The influence of weak and strong solid wall boundary conditions on the convergence to steady-state of the Navier-Stokes equations. In Proc. 19th AIAA CFD Conference, number 2009-3551 in Conference Proceeding Series. AIAA, 2009.

[17] X. Huan, J.E. Hicken, and D.W. Zingg. Interface and Boundary Schemes for High-Order Methods. In the 39th AIAA Fluid Dynamics Conference, AIAA Paper No. 2009-3658, San Antonio, USA, 22-25 June 2009.

[18] Mark H. Carpenter, Jan Nordström, and David Gottlieb. A Stable and Conservative Interface Treatment of Arbitrary Spatial Accuracy. Journal of Computational Physics, 148(2):341 - 365, 1999.

[19] Sofia Eriksson, Qaisar Abbas, and Jan Nordström. A stable and conservative method for locally adapting the design order of finite difference schemes. Journal of Computational Physics, In Press, Accepted Manuscript:-, 2010.

\section{A Double roots}

When determining the solutions to the recurrence relation from the Laplace transformed scheme in the interior, one has to be careful with double roots of the characteristic equation. Due to the ansatz, false roots might be introduced and it is necessary to confirm whether or not these roots belong to the spectrum. 
The characteristic equation (19) has double roots for $\tilde{s}=-4$ and $\tilde{s}=0$. The solutions are

$$
\kappa=-1, \quad \kappa=1
$$

respectively. The general solution to the recurrence relation is then

$$
\hat{v}_{i}=\left(c_{1}+c_{2} i\right) \kappa^{i} .
$$

We assume that the general solution (83) is valid for $i=1, \ldots N-1$ and insert into the modified boundary equations to get the matrix equation $E(s, \kappa) c=0$ for the unknowns $c=\left[\hat{v}_{0}, c_{1}, c_{2}, \hat{v}_{N}\right]^{T}$ where

$E(s, \kappa)=\left[\begin{array}{cccc}\tilde{s}+2 & 0 & 0 & 0 \\ -2 & ((\tilde{s}+2)-\kappa) \kappa & ((\tilde{s}+2)-2 \kappa) \kappa & 0 \\ 0 & ((\tilde{s}+2) \kappa-1) \kappa^{N-2} & ((\tilde{s}+2)(N-1) \kappa-(N-2)) \kappa^{N-2} & -2 \\ 0 & 0 & 0 & \tilde{s}+2\end{array}\right]$.

By inserting $s=-4$ and $\kappa=-1$ into (84) we get $\operatorname{det}(E(s, \kappa))=4 N(-1)^{N} \neq 0$. By inserting $\tilde{s}=0$ and $\kappa=1$ into (84) we get $\operatorname{det}(E(s, \kappa))=4 N \neq 0$. Hence neither $\tilde{s}=-4$ nor $\tilde{s}=0$ is a part of the spectrum. 